\title{
Tensor Estimation for Double-Pulsed Diffusional Kurtosis Imaging
}

Calvin B. Shaw, ${ }^{1,2}$ Edward S. Hui, ${ }^{3}$ Joseph A. Helpern, ${ }^{1,2,4,5}$ and Jens H. Jensen, ${ }^{1,2^{*}}$

${ }^{1}$ Center for Biomedical Imaging, Medical University of South Carolina, Charleston, South Carolina, USA.

${ }^{2}$ Department of Radiology and Radiological Science, Medical University of South Carolina, Charleston, South Carolina, USA.

${ }^{3}$ Department of Diagnostic Radiology, Li Ka Shing Faculty of Medicine, The University of Hong Kong, Pokfulam, Hong Kong SAR, China.

${ }^{4}$ Department of Neuroscience, Medical University of South Carolina, Charleston, South Carolina, USA.

${ }^{5}$ Department of Neurology, Medical University of South Carolina, Charleston, South Carolina, USA.

*Corresponding author at:

Center for Biomedical Imaging, Department of Radiology and Radiological Science, Medical University of South Carolina, 96 Jonathan Lucas, MSC 323, Charleston, SC 29425-0323, USA E-mail address: jense@musc.edu

Telephone: (843)876-2467

Grant sponsor: The Litwin Foundation

Grant number: N/A

Word count: 6468 


\section{ABSTRACT:}

Double-pulsed diffusional kurtosis imaging (DP-DKI) represents the double diffusion encoding (DDE) MRI signal in terms of six-dimensional (6D) diffusion and kurtosis tensors. Here a method for estimating these tensors from experimental data is described. A standard numerical algorithm for tensor estimation from conventional (i.e., single diffusion encoding) diffusional kurtosis imaging (DKI) data is generalized to DP-DKI. This algorithm is based on a weighted least squares (WLS) fit of the signal model to the data combined with constraints designed to minimize unphysical parameter estimates. The numerical algorithm then takes the form of a quadratic programming problem. The principal change required to adapt the conventional DKI fitting algorithm to DP-DKI is replacing the three-dimensional diffusion and kurtosis tensors with the 6D tensors needed for DP-DKI. In this way, the 6D diffusion and kurtosis tensors for DP-DKI can be conveniently estimated from DDE data by using constrained WLS, providing a practical means for condensing DDE measurements into well-defined mathematical constructs that may be useful for interpreting and applying DDE MRI. Data from healthy volunteers for brain are used to demonstrate the DP-DKI tensor estimation algorithm. In particular, representative parametric maps of selected tensor-derived rotational invariants are presented.

Keywords: double diffusion encoding, kurtosis, microscopic diffusion anisotropy, tensor, least squares, brain, DKI, MRI

Abbreviations used: $D D E$, double diffusion encoding; $D P-D K I$, double pulsed diffusional kurtosis imaging; DKI, diffusional kurtosis imaging; dMRI, diffusion MRI; DTI, diffusion tensor imaging; $D W I$, diffusion weighted image; $F A_{3 D}$, fractional anisotropy for three-dimensional diffusion tensor; $F A_{6 D}$, fractional anisotropy for six-dimensional diffusion tensor; $K F A_{3 D}$, kurtosis fractional anisotropy for three-dimensional kurtosis tensor; $K F A_{6 D}$, kurtosis fractional anisotropy for six-dimensional kurtosis tensor; $M G C$, multiple Gaussian compartment; ROI, region of interest; $S D E$, single diffusion encoding; $6 D$, six-dimensional; $3 D$, three-dimensional; $W L S$, weighted least squares; $\mu F A$, microscopic fractional anisotropy. 


\section{INTRODUCTION}

Double diffusion encoding (DDE) MRI is an emerging technique that differs from conventional single diffusion encoding (SDE) MRI by utilizing two diffusion wave vectors (or qvectors) per signal excitation rather than just one $(1,2)$. This allows additional information regarding the water diffusion dynamics to be obtained that relates to the conditional probability of a first diffusion displacement being followed by a second diffusion displacement, to which SDE MRI is completely insensitive. As a consequence, several rotationally invariant diffusion metrics may be derived with DDE MRI, which are impossible to measure with SDE MRI (3-7). Some of these pertain to microscopic diffusion anisotropy, which may be large even in tissues for which macroscopic measures, such as the fractional anisotropy, are small or vanish.

Most prior studies applying DDE MRI have employed either phantoms or biological specimens in order to develop and validate this method $(1,4,8,9)$, although application to in vivo brain has been reported in several recent studies (5-7,10,11). Most of these have focused on the technical aspects of DDE MRI, reflecting the challenges associated with this approach. However, Lawrenz and coworkers (11) report that one index of microscopic diffusion anisotropy is more sensitive in detecting differences in brain tissue microstructure related to aging than the conventional fractional anisotropy obtained with SDE MRI. The feasibility of utilizing DDE MRI for human imaging is expected to increase in the coming years with the growing availability of clinical scanners with gradient strengths of $80 \mathrm{mT} / \mathrm{m}$ and higher (5).

For SDE MRI, the cumulant expansion provides a natural approach for representing the diffusion-weighted signal and is the basis for commonly used analysis methods, including diffusion tensor imaging (DTI) (13) and diffusional kurtosis imaging (DKI) $(14,15)$. The extension of the cumulant expansion to DDE MRI was first considered by Jespersen and Buhl $(16,17)$. This leads to a series for the logarithm of the DDE signal in terms of powers of the two q-vectors. With mild assumptions, this series contains only even order terms. In order to accommodate diffusion anisotropy, each term of the series involves tensors, which encapsulate the essential diffusion information that can be gleaned from DDE. The leading (second order) term includes two tensors-the conventional diffusion tensor and a displacement correlation tensor (16). The next term is of fourth order and is composed of three tensors, the conventional kurtosis tensor together with two tensors that encode information unique to DDE (17). In this way, DDE MRI data can be systematically distilled into well-defined mathematical constructs. In 
principle, the cumulant expansion approach is only applicable when the diffusion weighting is not too strong, but as experience with SDE suggests, it is likely to be relevant for many experiments of practical interest.

Recently, it has been shown that the cumulant expansion for DDE can be reformulated in terms of six-dimensional (6D) tensors $(5,7,18)$. This simplifies the expansion considerably in that only a single tensor occurs with each term. Moreover, these 6D tensors are direct analogues of the three-dimensional (3D) tensors that arise in the cumulant expansion for SDE MRI. This allows the cumulant expansion for DDE to be analyzed in much the same manner as is routinely done for SDE.

In prior work, we demonstrated how to apply this $6 \mathrm{D}$ approach for the analysis of the DDE MRI signal to in vivo dMRI data from brain $(5,7)$. As is typical for SDE, we truncated the cumulant expansion after the fourth order term. For SDE, this truncation corresponds to DKI $(14,15)$, and so we refer to the DDE generalization as double-pulsed DKI (DP-DKI). In order to simplify the mathematical considerations, we extended, for these initial papers, the "fast kurtosis imaging" method of Hansen and coworkers (19) to DDE. This allowed us to derive a single novel rotational invariant without explicitly constructing the full 6D kurtosis tensor, and it also substantially simplified the data acquisition protocol. However in doing so, much of the information contained in the 6D kurtosis tensor is neglected.

The purpose of this paper is to describe and demonstrate a method of estimating the full 6D diffusion and kurtosis tensors from DDE data. By virtue of the 6D cumulant expansion for DDE being a straightforward extension of the conventional 3D cumulant expansion for SDE, we are able to adapt an existing method that has been frequently applied for standard (SDE) DKI (20). In essence, one need only replace the 3D tensors of standard DKI with their 6D generalizations. While this is relatively straightforward from a coding perspective, it is challenging computationally due to the larger size of the 6D tensors. Specifically, the $6 \mathrm{D}$ diffusion tensor has 12 independent components, compared to 6 in 3D, and the 6D kurtosis tensor has 66 independent components, compared to 15 in 3D. This higher number of independent components also necessitates that data for at least 66 diffusion encoding directions be obtained, which impacts the image acquisition time.

At the core of our method is a weighted least squares (WLS) fit of the signal model to the data, which is based on one of the several methods previously proposed for standard DKI 
(20,21). The WLS approach is chosen because of its simplicity, efficiency, and numerical robustness, which are key considerations in view of the large number of independent parameters needed to fully specify the 6D diffusion and kurtosis tensors. In order to minimize the occurrence of unphysical parameter values (e.g., negative diffusivities), the WLS fitting is augmented by a set of constraints that render the computational task into a quadratic programming problem, which is amenable to solution via standard techniques (22).

DDE data in brain from two healthy volunteers obtained with a $3 \mathrm{~T}$ clinical scanner are used to demonstrate the method. Here we consider just the calculation of a few of the simplest possible rotational invariants that can be derived from the 6D tensors, even though they represent only a fraction of the total information content. Our goal is to give preliminary examples of parametric maps based on these diffusion metrics rather than to infer strong conclusions regarding brain tissue microstructure. Nonetheless, we do briefly consider the physical significance of the considered invariants.

\section{THEORY AND METHODS}

\section{Cumulant Expansion for DDE Signal}

The DDE signal magnitude $S$ can be regarded as a function of two 3D q-vectors, $\mathbf{q}_{1} \equiv \gamma \delta g_{1} \mathbf{n}_{1} / 2 \pi$ and $\mathbf{q}_{2} \equiv \gamma \delta g_{2} \mathbf{n}_{2} / 2 \pi$, where $\gamma$ is the proton gyromagnetic ratio, $\delta$ is the diffusion gradient pulse duration for the DDE sequence, $g_{1}$ and $\mathbf{n}_{1}$ are the gradient amplitude and direction for the first block of diffusion encoding gradients, and $g_{2}$ and $\mathbf{n}_{2}$ are the gradient amplitude and direction for the second block of diffusion encoding gradients $(1,2)$. The gradient directions are normalized so that $\left|\mathbf{n}_{1}\right|=\mathbf{n}_{2} \mid=1$. A cumulant expansion for $S$ in terms of $\mathbf{q}_{\mathbf{1}}$ and $\mathbf{q}_{2}$ was originally derived by Jespersen and Buhl $(16,17)$. In order to simplify this expansion, Jensen and coworkers $(5,18)$ proposed concatenating these two vectors into a single 6D vector $\tilde{\mathbf{q}} \equiv\left(\mathbf{q}_{1}, \mathbf{q}_{2}\right)$. Here and below we use a tilde to indicate $6 \mathrm{D}$ objects. The cumulant expansion can then be written as

$$
\ln [S(\tilde{\mathbf{q}})]=\ln [S(0)]-\frac{\tilde{b}}{\tilde{q}^{2}} \sum_{\alpha, \beta=1}^{6} \tilde{q}_{\alpha} \tilde{q}_{\beta} \tilde{D}_{\alpha \beta}+\frac{(\tilde{b} \bar{D})^{2}}{6 \tilde{q}^{4}} \sum_{\alpha, \beta, \gamma, \delta=1}^{6} \tilde{q}_{\alpha} \tilde{q}_{\beta} \tilde{q}_{\gamma} \tilde{q}_{\delta} \tilde{W}_{\alpha \beta \gamma \delta}+O\left(\tilde{q}^{6}\right),
$$


where $\tilde{D}_{\alpha \beta}$ is a component of the 6D diffusion tensor, $\tilde{\mathbf{D}}$, and $\tilde{W}_{\alpha \beta \gamma \delta}$ is a component of the 6D kurtosis tensor, $\tilde{\mathbf{W}}$. In addition, $\tilde{q} \equiv|\tilde{\mathbf{q}}|, \tilde{q}_{\alpha}$ indicates a component of $\tilde{\mathbf{q}}, \tilde{b}$ is the $6 \mathrm{D}$ b-value, and $\bar{D}$ is the conventional mean diffusivity. The $6 \mathrm{D}$ b-value is defined by

$$
\tilde{b} \equiv(2 \pi \tilde{q})^{2}\left(\Delta-\frac{\delta}{3}\right),
$$

where rectangular gradient pulses are assumed and $\Delta$ is time between the centers of the two lobes for each block of diffusion encoding gradients $(5,7,18)$. We refer to $\Delta$ as the diffusion time, although some authors use this term instead for $\Delta-\delta / 3$. The validity of Equation [1] relies on the narrow pulse condition in which $\delta$ is assumed to be small (18).

Note that $\tilde{b}$ is proportional to $\tilde{q}^{2}$ so that the prefactors before the two sums in Equation [1] are independent of $\tilde{\mathbf{q}}$. By introducing the $6 \mathrm{D}$ diffusion encoding direction vector, $\tilde{\mathbf{n}} \equiv \tilde{\mathbf{q}} / \tilde{q}$, Equation [1] may be recast as

$$
\ln [S(\widetilde{\mathbf{q}})]=\ln [S(0)]-\tilde{b} \sum_{\alpha, \beta=1}^{6} \tilde{n}_{\alpha} \tilde{n}_{\beta} \widetilde{D}_{\alpha \beta}+\frac{(\tilde{b} \bar{D})^{2}}{6} \sum_{\alpha, \beta, \gamma, \delta=1}^{6} \tilde{n}_{\alpha} \tilde{n}_{\beta} \tilde{n}_{\gamma} \tilde{n}_{\delta} \tilde{W}_{\alpha \beta \gamma \delta}+O\left(\tilde{b}^{3}\right),
$$

where $\tilde{n}_{\alpha}$ represents a component of $\tilde{\mathbf{n}}$. Note that $|\tilde{\mathbf{n}}|=1$ by definition. Equation [3] is the 6D generalization of the familiar 3D cumulant expansion upon which standard DKI is based (9). An alternative form for Equation [3] is

$$
\ln [S(\tilde{\mathbf{q}})]=\ln [S(0)]-\tilde{b} \tilde{D}(\tilde{\mathbf{n}})+\frac{1}{6}[\tilde{b} \tilde{D}(\tilde{\mathbf{n}})]^{2} \tilde{K}(\tilde{\mathbf{n}})+O\left(\tilde{b}^{3}\right),
$$

where

$$
\tilde{D}(\tilde{\mathbf{n}}) \equiv \sum_{\alpha, \beta=1}^{6} \tilde{n}_{\alpha} \tilde{n}_{\beta} \widetilde{D}_{\alpha \beta}
$$

is the $6 \mathrm{D}$ diffusivity for the direction $\tilde{\mathbf{n}}$ and

$$
\tilde{K}(\tilde{\mathbf{n}}) \equiv\left[\frac{\bar{D}}{\widetilde{D}(\tilde{\mathbf{n}})}\right]^{2} \sum_{\alpha, \beta, \gamma, \delta=1}^{6} \tilde{n}_{\alpha} \tilde{n}_{\beta} \tilde{n}_{\gamma} \tilde{n}_{\delta} \tilde{W}_{\alpha \beta \gamma \delta}
$$

is the corresponding $6 \mathrm{D}$ diffusional kurtosis. Since $\tilde{b}$ and $\tilde{\mathbf{n}}$ uniquely determine $\tilde{\mathbf{q}}$ (for given $\Delta$ and $\delta$ ), one may equally well write $S(\tilde{b}, \tilde{\mathbf{n}})$ instead of $S(\tilde{\mathbf{q}})$ in Equations [1], [3], and [4].

It is important to appreciate that the 6D cumulant expansion for the DDE signal holds generically for all diffusive media (just as the 3D cumulant expansion does for SDE), save a few 
exotic cases that are likely of little relevance to diffusion MRI (dMRI) in biological tissues. In other words, Equations [1], [3], and [4] describe the physics of the diffusion process in general, without relying on assumptions about the detailed properties of a tissue. This sort of signal model should be distinguished from those based on explicit models of the diffusion dynamics in a particular tissue (i.e., dMRI tissue models). Moreover, the two tensors $\widetilde{\mathbf{D}}$ and $\tilde{\mathbf{W}}$ encapsulate all the information contained in the DDE signal up to order $\tilde{b}^{2}$ and thereby provide a complete description of DDE phenomena for low diffusion weightings.

As previously noted, the 6D diffusion tensor has 12 independent components. Of these, 6 coincide with those of the 3D diffusion tensor $\mathbf{D}$, evaluated at the diffusion time $\Delta$. The other 6 also depend on $\mathbf{D}$, but for different diffusion times. More specifically, we have $(5,16)$

$$
\tilde{\mathbf{D}}(\Delta, \tau)=\left(\begin{array}{cc}
\mathbf{D}(\Delta) & \mathbf{C}(\Delta, \tau) \\
\mathbf{C}(\Delta, \tau) & \mathbf{D}(\Delta)
\end{array}\right),
$$

where $\tau$ is the mixing time for the DDE sequence and

$$
\mathbf{C}(\Delta, \tau)=\frac{1}{2 \Delta}[(2 \Delta+\tau) \mathbf{D}(2 \Delta+\tau)+\tau \mathbf{D}(\tau)-2(\Delta+\tau) \mathbf{D}(\Delta+\tau)] .
$$

From Equation [8], it follows that $\mathbf{C}$ vanishes whenever the 3D diffusion tensor is independent of the diffusion time. If $\mathbf{D}$ has a weak time dependence, then Equation [8] implies

$$
\mathbf{C}(\Delta, \tau) \approx \Delta \cdot \mathbf{D}^{\prime}\left(\frac{3}{2}(\Delta+\tau)\right),
$$

where $\mathbf{D}^{\prime}(t) \equiv \partial \mathbf{D}(t) / \partial t$. Thus $\mathbf{C}$ is an approximate indicator of the time derivative of $\mathbf{D}$. A derivation of Equation [9] is given in Appendix A.

The 3D tensor $\mathbf{C}$ represents the additional information that DP-DKI provides beyond standard DKI to the leading order in the b-value, and it is equivalent to the displacement correlation tensor introduced by Jespersen and Buhl (16). However as is evident from Equation [8], this information can alternatively be derived from a set of three SDE experiments with diffusion times of $\tau, \Delta+\tau$, and $2 \Delta+\tau$. So in this sense, the information contained in $\widetilde{\mathbf{D}}$ is not unique to DDE, which is a special case of a more general theorem that the full time dependence of the 3D diffusion tensor is sufficient to predict the dMRI signal to the leading order in the diffusion weighting for arbitrary dMRI pulse sequences $(17,23)$. 
Of the 66 independent components for the $6 \mathrm{D}$ kurtosis tensor, 15 recapitulate the independent components of the 3D kurtosis tensor. The remaining 51 components represent information that can only be acquired with DDE (or more complicated pulse sequences). In contrast to the tensor $\mathbf{C}$, these additional components cannot be determined from any possible set of SDE experiments. Thus, it is only the terms from the cumulant expansion of order $\tilde{b}^{2}$ and higher that provide truly novel information vis-à-vis SDE MRI. An advantage of the DP-DKI approach is that the order $\tilde{b}^{2}$ information is fully contained within the 6D kurtosis tensor.

\section{Symmetry Properties}

The 6D diffusion tensor has 36 components, but because of symmetry properties only 12 of these are independent $(5,18)$. Similarly, the 6D kurtosis tensor has 1296 components, but symmetry reduces this to 66 that are independent.

For $\tilde{\mathbf{D}}$, the first symmetry is that the components $\widetilde{D}_{\alpha \beta}$ are invariant with respect to an interchange of the indices $\alpha$ and $\beta$. This is simply the statement that $\widetilde{\mathbf{D}}$ is a fully symmetric tensor, as is $\mathbf{D}$. An additional symmetry, which follows from time reversal and time translation invariance of the microscopic molecular dynamics (18), is

$$
\tilde{D}_{\alpha \beta}=\tilde{D}_{\alpha^{\prime} \beta^{\prime}} \text {, }
$$

where

$$
\alpha^{\prime}=1+[(\alpha+2) \bmod 6] \text { and } \beta^{\prime}=1+[(\beta+2) \bmod 6] .
$$

It is the combination of these interchange and time reversal symmetries that result in only 12 of the 36 components being independent.

For $\tilde{\mathbf{W}}$, the same two symmetries also apply. First, the 6D diffusion tensor is fully symmetric, just as for the 3D case. Second, the time invariances imply that

$$
\tilde{W}_{\alpha \beta \gamma \delta}=\tilde{W}_{\alpha^{\prime} \beta^{\prime} \gamma^{\prime} \delta^{\prime}},
$$

where in analogy to Equation [11],

$$
\gamma^{\prime}=1+[(\gamma+2) \bmod 6] \text { and } \delta^{\prime}=1+[(\delta+2) \bmod 6] \text {. }
$$

Again the combination of the two types of symmetries determines the number of independent components.

We also note that, as follows from Equation [7], 


$$
\tilde{D}_{i j}=D_{i j}
$$

where $D_{i j}$ represents a component of $\mathbf{D}$ and with the indices $i$ and $j$ ranging from 1 to 3 . Similarly,

$$
\tilde{W}_{i j k l}=W_{i j k l} \text {, }
$$

where $W_{i j k l}$ represents a component of the 3D kurtosis tensor $\mathbf{W}$ and with all the indices again ranging from 1 to 3. Hence, the 3D diffusion and kurtosis tensors are contained within the 6D tensors as subunits. Here and elsewhere Latin letters are used for indices that vary from 1 to 3, while Greek letters are used for indices running from 1 to 6.

\section{Rotational Invariants}

Typically, one is most often interested in quantities that are independent of the choice for the physical 3D coordinate system. These are the ordinary rotational invariants, which need only be unchanged with respect to a rotation in 3D. A quantity that is invariant with respect to 6D rotations is also a $3 \mathrm{D}$ invariant but the converse is not necessarily true.

The simplest rotational invariants for DP-DKI are linear forms in either $\widetilde{\mathbf{D}}$ or $\tilde{\mathbf{W}}$. For $\tilde{\mathbf{D}}$ , the linear invariants span a two-dimensional space. In order to devise a basis set for this space, we first define the $3 \mathrm{D}$ direction vector

$$
\mathbf{u}(\theta, \phi) \equiv \hat{\mathbf{x}} \sin (\theta) \cos (\varphi)+\hat{\mathbf{y}} \sin (\theta) \sin (\varphi)+\hat{\mathbf{z}} \cos (\theta)
$$

where $(\theta, \phi)$ are the standard spherical angles and $(\hat{\mathbf{x}}, \hat{\mathbf{y}}, \hat{\mathbf{z}})$ are Cartesian coordinate unit vectors. Note that Equation [16] implies that $|\mathbf{u}|=1$, so that $\mathbf{u}$ is also a unit vector. From $\mathbf{u}$, we can construct a pair of $6 \mathrm{D}$ direction vectors as

$$
\tilde{\mathbf{u}}^{ \pm}(\theta, \varphi)=\frac{1}{\sqrt{2}}[\mathbf{u}(\theta, \varphi), \pm \mathbf{u}(\theta, \varphi)]
$$

We then define the 6D mean parallel ( $\tilde{D}_{+}^{\dagger}$ ) and antiparallel ( $\tilde{D}_{-}^{\dagger}$ ) diffusivities by

$$
\tilde{D}_{ \pm}^{\dagger} \equiv \frac{1}{4 \pi} \int_{0}^{\pi} \sin (\theta) d \theta \int_{0}^{2 \pi} d \varphi \sum_{\alpha, \beta=1}^{6} \widetilde{D}_{\alpha \beta} \tilde{u}_{\alpha}^{ \pm}(\theta, \varphi) \tilde{u}_{\beta}^{ \pm}(\theta, \varphi),
$$

with $\tilde{u}_{\alpha}^{ \pm}$being a component of $\tilde{\mathbf{u}}^{ \pm}$. Since $\tilde{D}_{+}^{\dagger}$ and $\tilde{D}_{-}^{\dagger}$ are defined as integrals over all 3D diffusion directions, they are manifestly rotational invariants. After performing the integrals in Equation [18], this simplifies to 


$$
\tilde{D}_{ \pm}^{\dagger}=\frac{1}{3} \operatorname{tr}(\mathbf{D} \pm \mathbf{C})
$$

Clearly, we have

$$
\bar{D} \equiv \frac{1}{3} \operatorname{tr}(\mathbf{D})=\frac{1}{2}\left(\tilde{D}_{+}^{\dagger}+\tilde{D}_{-}^{\dagger}\right)
$$

and

$$
\bar{C} \equiv \frac{1}{3} \operatorname{tr}(\mathbf{C})=\frac{1}{2}\left(\widetilde{D}_{+}^{\dagger}-\widetilde{D}_{-}^{\dagger}\right)
$$

Thus any two of these four diffusivity invariants (i.e., $\tilde{D}_{+}^{\dagger}, \tilde{D}_{-}^{\dagger}, \bar{D}, \bar{C}$ ) can be used to calculate the others. Moreover, any rotationally invariant metric that is linear in $\tilde{\mathbf{D}}$ can be expressed as a linear combination of $\tilde{D}_{+}^{\dagger}$ and $\tilde{D}_{-}^{\dagger}$. Therefore, these two metrics form a basis set for linear rotational invariants derived from $\tilde{\mathbf{D}}$. Recalling that the $3 \mathrm{D}$ tensor $\mathbf{C}$ can be determined with SDE MRI, one sees that neither $\widetilde{D}_{+}^{\dagger}$ nor $\tilde{D}_{-}^{\dagger}$ is unique to DDE MRI. As follows from Equations [8], [20], and [21], $\tilde{D}_{+}^{\dagger}=\tilde{D}_{-}^{\dagger}=\bar{D}$ whenever the diffusion tensor $\mathbf{D}$ is independent of time.

For linear rotational invariants based on $\tilde{\mathbf{W}}$, we first have the 3D mean kurtosis tensor given by (19)

$$
\bar{W} \equiv \frac{1}{4 \pi} \int d \Omega_{n} \sum_{i, j, k, ;=1}^{3} W_{i j k l} n_{i} n_{j} n_{k} n_{l},
$$

where the integral is over all 3D directions and $n_{i}$ indicates the component of a 3D direction vector. After performing the integral, this simplifies to

$$
\bar{W}=\frac{1}{5}\left(W_{1111}+W_{2222}+W_{3333}+2 W_{1122}+2 W_{1133}+2 W_{2233}\right) .
$$

Similarly, the 6D mean kurtosis tensor is defined by (5)

$$
\widetilde{\widetilde{W}} \equiv \frac{1}{\pi^{3}} \int d \tilde{\Omega}_{\tilde{n}} \sum_{\alpha, \beta, \gamma, \delta=1}^{6} \tilde{W}_{\alpha \beta \gamma \delta} \tilde{n}_{\alpha} \tilde{n}_{\beta} \tilde{n}_{\gamma} \tilde{n}_{\delta},
$$

with the integral being over all 6D directions, which works out to

$$
\begin{aligned}
\tilde{\widetilde{W}}=\frac{1}{8}\left(\tilde{W}_{1111}+\tilde{W}_{2222}+\tilde{W}_{3333}+2 \tilde{W}_{1122}+2 \tilde{W}_{1133}+2 \tilde{W}_{2233}\right. \\
\left.+\tilde{W}_{1144}+\tilde{W}_{2255}+\tilde{W}_{3366}+2 \tilde{W}_{1155}+2 \tilde{W}_{1166}+2 \tilde{W}_{2266}\right) .
\end{aligned}
$$


In general, $\widetilde{\bar{W}}$ is not equal to $\bar{W}$, and it represents an independent metric $(5,7)$. We can also construct 6D mean parallel and anti-parallel kurtosis tensors as

$$
\tilde{W}_{ \pm}^{\dagger} \equiv \frac{1}{4 \pi} \int_{0}^{\pi} \sin (\theta) d \theta \int_{0}^{2 \pi} d \varphi \sum_{\alpha, \beta, \gamma, \delta=1}^{6} \tilde{W}_{\alpha \beta \gamma \delta} \tilde{u}_{\alpha}^{ \pm}(\theta, \varphi) \tilde{u}_{\beta}^{ \pm}(\theta, \varphi) \tilde{u}_{\gamma}^{ \pm}(\theta, \varphi) \tilde{u}_{\delta}^{ \pm}(\theta, \varphi) .
$$

After evaluating the integral, this takes the form

$$
\begin{aligned}
\tilde{W}_{ \pm}^{\dagger}= & \frac{1}{10}\left(\tilde{W}_{1111}+\tilde{W}_{2222}+\tilde{W}_{3333}+2 \tilde{W}_{1122}+2 \tilde{W}_{1133}+2 \tilde{W}_{2233}+3 \tilde{W}_{1144}+3 \tilde{W}_{2255}+3 \tilde{W}_{3366}+2 \tilde{W}_{1155}\right. \\
& +2 \tilde{W}_{1166}+2 \tilde{W}_{2266}+4 \tilde{W}_{1245}+4 \tilde{W}_{1346}+4 \tilde{W}_{2356} \pm 4 \tilde{W}_{1114} \pm 4 \tilde{W}_{2225} \pm 4 \tilde{W}_{3336} \pm 4 \tilde{W}_{1125} \pm 4 \tilde{W}_{1136} \\
& \left. \pm 4 \tilde{W}_{1224} \pm 4 \tilde{W}_{1334} \pm 4 \tilde{W}_{2236} \pm 4 \tilde{W}_{2335}\right) .
\end{aligned}
$$

The metrics $\bar{W}, \tilde{\bar{W}}, \tilde{W}_{+}^{\dagger}$, and $\tilde{W}_{-}^{\dagger}$ constitute a basis set for all linear rotational invariants derived from $\tilde{\mathbf{W}}$, so any other rotational invariant that is linear in $\tilde{W}$ can be expressed as a linear combination of $\bar{W}, \stackrel{\sim}{\bar{W}}, \tilde{W}_{+}^{\dagger}$, and $\tilde{W}_{-}^{\dagger}$. One specific linear combination of interest is the difference $\delta \bar{W} \equiv \bar{W}-\widetilde{\bar{W}}$, which is an index of microscopic diffusion anisotropy $(5,7)$.

The metric $\bar{W}$ can also be obtained with SDE MRI, but $\stackrel{\widetilde{W}}{,} \tilde{W}_{+}^{\dagger}$, and $\tilde{W}_{-}^{\dagger}$ cannot for arbitrary diffusion dynamics. However, one may show, as described in Appendix B, that R2,C24 $\tilde{W}_{ \pm}^{\dagger}=\bar{W}$ for multiple Gaussian compartment (MGC) models without inter-compartmental water exchange. Henceforth when we refer to MGC models, it is assumed that inter-compartmental water exchange is neglected, even though one may also formulate MGC models with exchange. We further assume, as is typically done, that the intra-compartmental diffusion tensors are independent of time.

Another natural set of rotational invariants to consider are the fractional anisotropies for $\mathbf{D}, \tilde{\mathbf{D}}, \mathbf{W}$, and $\tilde{\mathbf{W}}$. These can be written as

$$
\begin{aligned}
& \mathrm{FA}_{3 \mathrm{D}} \equiv \sqrt{\frac{3}{2}} \cdot \frac{\left\|\mathbf{D}-\bar{D} \mathbf{I}^{(2)}\right\|_{F}}{\|\mathbf{D}\|_{F}}, \\
& \mathrm{FA}_{6 \mathrm{D}} \equiv \sqrt{\frac{3}{2} \cdot \frac{\left\|\tilde{\mathbf{D}}-\bar{D} \tilde{\mathbf{I}}^{(2)}\right\|_{F}}{\|\tilde{\mathbf{D}}\|_{F}},} \\
& \mathrm{KFA}_{3 \mathrm{D}} \equiv \frac{\left\|\mathbf{W}-\bar{W} \mathbf{I}^{(4)}\right\|_{F}}{\|\mathbf{W}\|_{F}},
\end{aligned}
$$


and

$$
\mathrm{KFA}_{6 D} \equiv \frac{\left\|\tilde{\mathbf{W}}-\tilde{\widetilde{W}} \tilde{\mathbf{I}}^{(4)}\right\|_{F}}{\|\tilde{\mathbf{W}}\|_{F}}
$$

In Equations [28]-[31], $\|. . .\|_{F}$ indicates the Frobenius norm, while $\mathbf{I}^{(2)}, \tilde{\mathbf{I}}^{(2)}, \mathbf{I}^{(4)}$ and $\tilde{\mathbf{I}}^{(4)}$ are symmetric and isotropic tensors defined by their respective components:

$$
I_{i j}^{(2)}=\delta_{i j}, \widetilde{I}_{\alpha \beta}^{(2)}=\delta_{\alpha \beta}, I_{i j k l}^{(4)}=\frac{1}{3}\left(\delta_{i j} \delta_{k l}+\delta_{i k} \delta_{j l}+\delta_{i l} \delta_{j k}\right) \text {, and } \widetilde{I}_{\alpha \beta \gamma \delta}^{(4)}=\frac{1}{3}\left(\delta_{\alpha \beta} \delta_{\gamma \delta}+\delta_{\alpha \gamma} \delta_{\beta \delta}+\delta_{\alpha \delta} \delta_{\beta \gamma}\right) \text {, [32] }
$$

with $\delta_{i j}$ and $\delta_{\alpha \beta}$ being the Kronecker deltas in 3D and 6D, respectively. The $\mathrm{FA}_{3 \mathrm{D}}$ and $\mathrm{KFA}_{3 \mathrm{D}}$ are standard measures of diffusion anisotropy familiar from SDE MRI (24). The FA FD $_{\text {can also }}$ be determined with SDE MRI, if multiple diffusion times are employed in order to construct $\widetilde{\mathbf{D}}$ as discussed above. In contrast, the $\mathrm{KFA}_{6 \mathrm{D}}$ is a novel metric that encodes information specific to DDE MRI, and it should be a sensitive indicator of microscopic diffusion anisotropy, because any type of anisotropy that is encompassed by the fourth order term in the cumulant expansion of Eq. [1] will affect $\mathrm{KFA}_{6 \mathrm{D}}$. This is in contrast with $\delta \bar{W}$, which is only sensitive to a specific type of microscopic anisotropy (5,7). The kurtosis anisotropies of Equations [30] and [31] are indeterminate when the denominators vanish (as occurs for Gaussian diffusion). In these cases, the kurtosis anisotropies may be defined as zero.

The quantities given by Equations [19]-[21], [23], [25], [27], and [28]-[31] are invariant with respect to rotations in the physical 3D space. Of these, only $\bar{D}, \tilde{W}, \mathrm{FA}_{6 \mathrm{D}}$, and $\mathrm{KFA}_{6 \mathrm{D}}$ are also generally invariant for all rotations in the 6D extended space. However, in the special case of MGC models, FA $_{3 \mathrm{D}}$ is invariant under 6D rotations as well.

\section{WLS for DP-DKI}

There are a variety of methods for fitting signal models to dMRI data. Here we choose WLS for the sake of simplicity and numerical robustness. Since there are a total of $12+66=78$ independent parameters to be estimated, a robust method such as WLS has the important advantage, in comparison to many other methods, of avoiding the difficulties associated with multiple local minima. In addition, WLS is the basis of our publically available post-processing software for standard DKI (25). 
To define the WLS procedure, we suppose that experimental data for the DDE signal has been obtained for $\tilde{q}=0$ and for $M$ 6D q-vectors with $\tilde{q}>0$. If $\tilde{\mathbf{q}}_{m}$ is the $m$ th nonzero q-vector, let us introduce the one-dimensional array, $\mathbf{Y}$, with the $M$ components

$$
Y_{m} \equiv \ln \left[\frac{S\left(\tilde{\mathbf{q}}_{m}\right)}{S(0)}\right] .
$$

Each q-vector also has an associated b-value, $\widetilde{b}_{m}$, and an associated 6D diffusion direction, $\tilde{\mathbf{n}}_{m}$, with the components $\tilde{n}_{\alpha, m}$. After dropping the $O\left(\tilde{b}^{3}\right)$ terms, the cumulant expansion of Equation [3] can then be expressed as

$$
Y_{m}=-\tilde{b}_{m} \sum_{\alpha, \beta=1}^{6} \tilde{n}_{\alpha, m} \tilde{n}_{\beta, m} \tilde{D}_{\alpha \beta}+\frac{\tilde{b}_{m}^{2}}{6} \sum_{\alpha, \beta, \gamma, \delta=1}^{6} \tilde{n}_{\alpha, m} \tilde{n}_{\beta, m} \tilde{n}_{\gamma, m} \tilde{n}_{\delta, m} \tilde{H}_{\alpha \beta \gamma \delta} \text {, for } \quad m=1,2, \ldots, M,
$$

where

$$
\tilde{H}_{\alpha \beta \gamma \delta} \equiv \bar{D}^{2} \tilde{W}_{\alpha \beta \gamma \delta} .
$$

Equation [34] constitutes a set of $M$ linear equations for the tensor components $\widetilde{D}_{\alpha \beta}$ and $\tilde{H}_{\alpha \beta \gamma \delta}$. Since there are a total of 78 unknown parameters, we require $M \geq 78$ in order to have a unique solution. Once $\tilde{D}_{\alpha \beta}$ and $\tilde{H}_{\alpha \beta \gamma \delta}$ have been determined, then $\bar{D}$ is found from Equation [20] and the 6D kurtosis tensor is found by inverting Equation [35] to give $\tilde{W}_{\alpha \beta \gamma \delta}=\tilde{H}_{\alpha \beta \gamma \delta} / \bar{D}^{2}$.

As shown in Appendix C, the sums in Equation [34] can be written explicitly in terms of the 12 independent components for $\tilde{\mathbf{D}}$ and the 66 independent components for $\tilde{\mathbf{H}} \equiv \bar{D}^{2} \tilde{\mathbf{W}}$. These independent tensor components may be reorganized into a one-dimensional array, $\mathbf{X}$, with 78 components. Equation [34] can be then be written in the canonical form

$$
\mathbf{Y}=\mathbf{A X} \text {, }
$$

where $\mathbf{A}$ is a matrix with $M \times 78$ components. An explicit representation of $\mathbf{A}$ is given in Appendix D.

If Equation [36] is solved in the least squares sense, then one has

$$
\mathbf{X}=\left(\mathbf{A}^{\mathrm{T}} \mathbf{A}\right)^{-1} \mathbf{A}^{\mathrm{T}} \mathbf{Y},
$$

where $\left(\mathbf{A}^{\mathrm{T}} \mathbf{A}\right)^{-1} \mathbf{A}^{\mathrm{T}}$ is the pseudoinverse. However, in order to take into account that the standard deviation for a measurement of $Y_{m}$ scales approximately as $1 / S\left(\tilde{\mathbf{q}}_{m}\right)$, a better estimate for $\mathbf{X}$ comes from the WLS result of (26) 


$$
\mathbf{X}=\left(\mathbf{A}^{\mathrm{T}} \boldsymbol{\Lambda} \mathbf{A}\right)^{-1} \mathbf{A}^{\mathrm{T}} \boldsymbol{\Lambda} \mathbf{Y},
$$

where $\boldsymbol{\Lambda}$ is a $M \times M$ diagonal matrix with the nonzero components

$$
\Lambda_{m m}=\left[S\left(\widetilde{\mathbf{q}}_{m}\right)\right]^{2} .
$$

Here we have assumed that the standard deviation associated with $S(0)$ is negligible, which is the case if a sufficient number of acquisitions with $\tilde{q}=0$ are averaged.

\section{Constraints and Quadratic Programming}

Because of noise, motion, and imaging artifacts (e.g., Nyquist ghosting), the DDE signal may, in practice, have significant errors that can confound the accurate estimation of the $6 \mathrm{D}$ diffusion and kurtosis tensors, if WLS is implemented by applying Equation [38] directly. This problem can be mitigated by imposing constraints on the tensor components that help to keep these within physically acceptable ranges.

Here we adapt to DP-DKI constraints similar to those used for standard DKI (20). The first of these is

$$
\widetilde{D}\left(\tilde{\mathbf{n}}_{m}\right)>0,
$$

so that the $6 \mathrm{D}$ diffusivity is positive in each diffusion encoding direction. The second is that the $6 \mathrm{D}$ kurtosis is restricted to the range

$$
0<\tilde{K}\left(\tilde{\mathbf{n}}_{m}\right)<\frac{3}{\widetilde{b}_{\max } \tilde{D}\left(\tilde{\mathbf{n}}_{m}\right)},
$$

where $\widetilde{b}_{\max }$ is the maximum b-value for the data acquisition. This lower bound is rigorously true for any MGC model $(5,14,27)$ and is expected to hold in most biological tissues, such as brain. The upper bound guarantees that the solution corresponds to a monotonically decreasing DDE signal $(20,28)$, over the b-value range of the data acquisition, as is empirically observed for brain. However, the DDE signal is not necessarily monotonically decreasing for certain highly ordered diffusive media $(1,8)$, in which case the constraints of Eq. [41] may not be appropriate.

In order to impose these constraints, the array $\mathbf{X}$ can be obtained by solving the quadratic programming problem

$$
\begin{aligned}
& \text { Minimize } \quad\left|\boldsymbol{\Lambda}^{1 / 2}(\mathbf{A X}-\mathbf{Y})\right|^{2}, \\
& \text { obtained such that } \quad \mathbf{G X}<0,
\end{aligned}
$$


where $\mathbf{G}$ is a $2 M \times 78$ constant matrix defined in Appendix $\mathrm{D}$, the vertical lines in Equation [42] indicate the Euclidean norm, and Equation [43] is understood to apply on an element-wise basis. Without the constraints of Equation [43], the solution to Equation [42] is given precisely by Equation [38]. With the constraints, this becomes a convex quadratic programming problem that can be readily solved with established methods (22). Crucially, any local minimum will also be a global minimum, since the objective function is convex (29). Also for $M \geq 78$, we typically expect to have a unique solution (assuming the matrix $\mathbf{A}$ is well conditioned). The practical impact of imposing the constraints of Equation [43] is illustrated below under Results.

\section{Diffusion Encoding Directions}

For the matrix A to be well conditioned, at least 66 independent diffusion encoding directions must be employed $(5,7)$, although this by itself is not sufficient. Here we use 80 $\mathrm{R} 2, \mathrm{C} 27$ independent $6 \mathrm{D}$ directions so as to have an overdetermined set of equations, which can help to reduce deleterious effects associated with noise and artifacts. For the first 21 of these directions, we choose those needed for the fast kurtosis approach, which has been previously described $(5,7)$. The advantage of this is that it permits an independent fast kurtosis analysis of the data to be performed, which can provide a useful consistency check. The other 59 directions are selected to minimize the condition number of the matrix $\mathbf{A}$ for the selected set of $6 \mathrm{D}$ b-values, similar to what has sometimes been done with DTI (30), in order to improve robustness with respect to experimental noise. For the experiments reported on here, the DDE MRI signal is sampled for both $\tilde{b}=1000 \mathrm{~s} / \mathrm{mm}^{2}$ and $\tilde{b}=2200 \mathrm{~s} / \mathrm{mm}^{2}$ in each of the 80 diffusion encoding directions. Thus we have $M=80+80=160$, which is just over twice the minimum of 78 q-space points needed for a unique solution to Equations [42] and [43]. To minimize the condition number of A the extra 59 directions were selected from an ensemble of 10000 randomly generated sets of encoding directions. This optimal set of 21+59 directions was used for all of the experimental measurements and is listed in Table 1 of Appendix E.

\section{Data Acquisition}

DP-DKI datasets were acquired from two healthy volunteers, both 23 yrs of age, using a protocol approved by the Institutional Review Board of the University of Hong Kong under informed consent. All data were obtain on a 3 T whole-body MR scanner (Achieva TX, Philips 
Healthcare, Best, The Netherlands) with a maximum gradient amplitude of $80 \mathrm{mT} / \mathrm{m}$ using an eight channel receive only head coil. A spin-echo echo-planar-imaging custom DDE pulse sequence was employed, as previously described (7). Diffusion weighted images (DWIs) were acquired with 3 6D b-values $\left(0,1000,2200 \mathrm{~s} / \mathrm{mm}^{2}\right)$, as defined by Equation [2], along 80 6D diffusion encoding directions (Appendix E) for 8 slices with fat suppression. This choice of 6D b-values is comparable to that of our prior studies (5,7). The diffusion time ( $\Delta$ ) was $26.3 \mathrm{~ms}$, the pulse duration $(\delta$ ) was $13.6 \mathrm{~ms}$, and the diffusion mixing time $(\tau)$ was $30.6 \mathrm{~ms}$. Other key scan parameters were TR/TE $=5500 / 115 \mathrm{~ms}$, field-of-view $=230 \times 230 \mathrm{~mm}^{2}$, acquisition matrix $=$ $80 \times 80$, slice thickness $=5 \mathrm{~mm}$ with no gap, receiver bandwidth $=3657 \mathrm{~Hz} /$ pixel and a SENSE factor of two along the phase encoding direction (anterior-posterior). For the nonzero b-values, a total of two signal acquisitions for each slice and diffusion encoding direction were obtained, while 17 acquisitions were collected with $\tilde{b}=0$ (b0 images). The total acquisition time was approximately $32 \mathrm{~min}$.

\section{Data Analysis}

All images for each subject were co-registered to the first b0 image acquired using SPM8 $\mathrm{R} 2, \mathrm{C} 32$ (Wellcome Trust Center for Neuroimaging, London, UK). An average DP-DKI dataset was created by averaging all corresponding b0 images and DWIs, which was then processed with custom scripts implemented in MATLAB (Math Works, Natick, MA). Thus, for each subject, 17 b0 images were averaged, and two images for each combination of diffusion encoding direction and nonzero b-value were averaged. The DWIs were filtered for Gibbs ringing artifact and noise using a Gaussian kernel with a full width at half maximum of $3.375 \mathrm{~mm}$, after which a constrained WLS algorithm was used to estimate the 6D tensors. The diffusion and kurtosis tensors were computed on a voxel-by-voxel basis to obtain the parametric maps for $\bar{D}, \bar{C}, \tilde{D}_{ \pm}^{\dagger}$, $\bar{W}, \tilde{\bar{W}}, \tilde{W}_{ \pm}^{\dagger}, \mathrm{FA}_{3 \mathrm{D}}, \mathrm{FA}_{6 \mathrm{D}}, \mathrm{KFA}_{3 \mathrm{D}}, \mathrm{KFA}_{6 \mathrm{D}}$. All calculations were carried out on a Dell Precision workstation equipped with a $2.27 \mathrm{GHz}$ Pentium Xeon processor. The computational time for a single dataset was $790 \mathrm{~s}$.

In addition to $\mathrm{KFA}_{6 \mathrm{D}}$, there are several other ways to quantify microscopic diffusion anisotropy in brain $(3-7,31,32)$, some of which are related. For example, the microscopic 
fractional anisotropy $(\mu \mathrm{FA})(31,32)$ has a close connection to the rotational invariant $\delta \bar{W} \equiv \bar{W}-\tilde{\bar{W}}$. Specifically, for MGC models, one may show that (7)

$$
\mu \mathrm{FA}=\sqrt{\frac{3}{2}}\left(1+\frac{9 \bar{D}^{2}}{9 \delta^{2} \lambda+20 \bar{D}^{2} \delta \bar{W}}\right)^{-\frac{1}{2}},
$$

where, $\delta^{2} \lambda$ is the variance of the diffusion tensor eigenvalues and is simply obtained from

$$
\delta^{2} \lambda=\frac{2 \bar{D}^{2} \mathrm{FA}_{3 \mathrm{D}}^{2}}{3-2 \mathrm{FA}_{3 \mathrm{D}}^{2}} .
$$

As a reference of comparison, we also used Equation [44] to estimate $\mu \mathrm{FA}$ along with other measures of diffusion anisotropy.

\section{RESULTS}

Figure 1 shows the $\bar{D}, \bar{C}, \widetilde{D}_{+}^{\dagger}$, and $\tilde{D}_{-}^{\dagger}$ maps obtained using the WLS algorithm for the two subjects, with three representative axial slices for each metric. Note that $\bar{C}$ is very small within the brain parenchyma so that $\bar{D} \approx \widetilde{D}_{+}^{\dagger} \approx \widetilde{D}_{-}^{\dagger}$, which is consistent with a 3D mean diffusivity that varies weakly with time over time scales comparable to $3(\Delta+\tau) / 2(\approx 85 \mathrm{~ms}$ for our experiment), as suggested by Equation [9].

For the same subjects and slices, Figure 2 gives the corresponding maps of the linear kurtosis metrics $\bar{W}, \tilde{\bar{W}}, \tilde{W}_{+}^{\dagger}$, and $\tilde{W}_{-}^{\dagger}$, along with $\delta \bar{W} \equiv \bar{W}-\tilde{\bar{W}}$. Although the maps of these four principal measures are all similar, clear differences are apparent between each of them. As has been previously discussed, $\delta \bar{W}$ is an index of microscopic diffusion anisotropy, while the fact that $\bar{W} \neq \tilde{W}_{+}^{\dagger} \neq \tilde{W}_{-}^{\dagger}$ suggests the water diffusion dynamics is not fully consistent with an MGC model, at least in certain brain regions. However, some of these differences could also be due to estimation errors.

Maps for the fractional anisotropies of $\mathrm{FA}_{3 \mathrm{D}}, \mathrm{FA}_{6 \mathrm{D}}, \mathrm{KFA}_{3 \mathrm{D}}, \mathrm{KFA}_{6 \mathrm{D}}$ and $\mu \mathrm{FA}$ are displayed in Figure 3. The $\mathrm{FA}_{3 \mathrm{D}}$ and $\mathrm{FA}_{6 \mathrm{D}}$ maps are nearly indistinguishable, which is a consequence of the fact that $\bar{C}$ is close to zero. In contrast, $\mathrm{KFA}_{3 \mathrm{D}}$ and $\mathrm{KFA}_{6 \mathrm{D}}$ are quite different, reflecting the novel information provided by $\mathrm{KFA}_{6 \mathrm{D}}$. Qualitatively, the $\mathrm{KFA}_{6 \mathrm{D}}$ map is 
seen to be similar to the $\mu \mathrm{FA}$ map. However, these two metrics have an important conceptual distinction in that $\mathrm{KFA}_{6 \mathrm{D}}$ is not tied to a specific model of the diffusion dynamics, unlike $\mu \mathrm{FA}$ which is only well-defined for MGC models.

In order to illustrate the effect of applying the constraints of Equation [43], we also performed a calculation of the diffusion metrics without these constraints being imposed. In most voxels, we find that this makes little or no difference. However, the maps for the linear kurtosis

metrics calculated without the constraints do possess several hypointense regions that are $\mathrm{R} 2, \mathrm{C} 37$ lessened or removed when the constraints are applied. This is demonstrated in Figure 4 for one subject and is similar to the effect of imposing constraints with standard SDE DKI (20). The artifacts that the constraints help to attenuate may be due to a variety of factors such as signal noise, motion, Nyquist ghosting, Gibbs ringing, thermal drift, and incomplete fat suppression.

For the vast majority of voxels, the signal model of Equation [34] is found to provide a close fit to the data. This is a not a trivial result as in each voxel there are 160 data points (i.e., $Y_{m}$ for $\left.m=1, \ldots, 160\right)$ but only 78 unknowns for the diffusion and kurtosis tensors. Examples of the fits for three selected regions of interest (ROIs) are given in Figure 5. Each ROI is a square containing 4 voxels on a single slice. For other choices of the 6D b-values, the quality of the fits would not necessarily be as good.

\section{DISCUSSION}

The analysis of DDE MRI data can be complicated due to the rich array of information that this technique provides. Accordingly, a variety of related methods have been previously proposed for this purpose $(1-7,16-18,33)$. Our present DP-DKI formulation in terms of 6D diffusion and kurtosis tensors demonstrates that DDE MRI can be analyzed in a manner analogous to that conventionally utilized with standard DKI. We believe this has substantial conceptual advantages, even if the mathematical details may seem baroque. In essence, the DPDKI formulation of DDE MRI follows the same fundamental logic of DTI and DKI that has proven successful for SDE MRI. The primary goal of this paper is to describe in detail a practical approach for performing DP-DKI calculations.

We have chosen to employ a constrained WLS algorithm because of its simplicity and numerical robustness. Constrained WLS is also frequently used for standard DKI, and the generalization to DP-DKI is relatively straightforward. The essential change is extending the 
diffusion and kurtosis tensors from 3D to 6D. As a consequence, the number of unknowns increases from 21 to 78 and the minimum number of diffusion encoding directions increases from 15 to 66. This means both a greater data acquisition burden and a more challenging numerical analysis. On human scanners, the quality of the data benefits from using systems with strong gradients having maximum magnitudes of $80 \mathrm{mT} / \mathrm{m}$ or higher, since this allows for much shorter echo times in comparison to systems with the more common gradient strength limit of about $40 \mathrm{mT} / \mathrm{m}$.

The 80 6D diffusion encoding directions listed in Table 1 of Appendix E were utilized primarily for reasons of expediency. The first 21 directions are identical to those of the fast kurtosis approach $(5,7)$. This allows some of the rotational invariants $(\bar{D}, \bar{W}, \widetilde{\bar{W}})$ to be determined directly without first explicitly calculating $\tilde{\mathbf{D}}$ and $\tilde{\mathbf{W}}$, which thereby provides a useful means of checking the results of the full WLS analysis. The remaining 59 directions were found by minimizing the condition number for the matrix $\mathbf{A}$. While this method of selecting the diffusion encoding directions is effective (30), it is likely not ideal, and we expect that improved sets of directions are possible.

The large number of independent components $(12+66=78)$ for the $6 \mathrm{D}$ diffusion and kurtosis tensors means that they can be used to construct several independent rotationally invariant quantities. Here we have only considered the simplest of these, which are those linear in either $\tilde{\mathbf{D}}$ or $\tilde{\mathbf{W}}$ and the fractional anisotropies for $\mathbf{D}, \mathbf{W}, \tilde{\mathbf{D}}$ and $\tilde{\mathbf{W}}$. In analogy with standard DKI, we conjecture that these most basic diffusion metrics are likely to represent, in practice, much of the useful information that can be obtained with DP-DKI. Moreover, we R2,C11 remind the reader that only those metrics related to the $6 \mathrm{D}$ kurtosis tensor $\tilde{\mathbf{W}}$ provide information that is inaccessible with SDE MRI. The 6D kurtosis fractional anisotropy, KFA ${ }_{6 \mathrm{D}}$, strikes us as a particularly promising measure for quantifying diffusion anisotropy (including microscopic anisotropy), although future work would be needed to verify this.

An important advantage of defining metrics for DDE MRI directly in terms of the 6D diffusion and kurtosis tensors is that this endows them with well-defined physical meanings independent of any particular model for the diffusion dynamics. This is in contrast with some other proposed metrics whose definitions rely on a priori assumptions $(3,4,6,7,31,32)$. For example, the usual definition of $\mu \mathrm{FA}(31,32)$ assumes an MGC model and is therefore not 
applicable to arbitrary diffusion dynamics. Nevertheless, for MGC models, one may directly relate the basic metrics we have considered here for DP-DKI to the $\mu \mathrm{FA}$, as demonstrated by Equation [44]. As another example, one may readily show (e.g., see equation A6 of Ref. 5) that the "isotropic kurtosis" defined by Szczepankiewicz and coworkers (34) is equivalent to $(8 \tilde{\bar{W}}-5 \bar{W}) / 3$ for any MGC model. In this way, DP-DKI includes other approaches as special cases. It can be noted in passing that for MGC models the 6D kurtosis tensor has only 21 independent components, which is less than one third of the number allowed in general.

Although our primary focus has been on the practical aspects of calculating the $6 \mathrm{D}$ diffusion and kurtosis tensors, the preliminary results presented here do lend themselves to some initial observations. First, for the diffusion and mixing times used in our DDE pulse sequence, the metric $\bar{C}$ is quite small in comparison to $\bar{D}$ (see Figure 1) in most brain tissue, implying R2,C13 that the mean diffusivity is not strongly time dependent. This is largely consistent with prior work in which the time dependence of the diffusion tensor in brain is measured directly (35). Another potentially significant finding is the fact that the $\bar{W}, \tilde{W}_{+}^{\dagger}$, and $\tilde{W}_{-}^{\dagger}$ maps have noticeable differences. The most straightforward interpretation is that this represents a departure from the predictions of MGC models, which require these three quantities to be identical. Since MGC models are widely applied in the modeling of dMRI data (36), this could have important implications for the accuracy of such models. However, the observed differences might also be partly the result of systematic errors in the estimation of the linear 6D kurtosis metrics due either

to the effect of truncating the cumulant expansion at the order $\tilde{b}^{2}$ term (see Equation [34]) or to imaging artifacts (e.g., Nyquist ghosting). Nonetheless, DP-DKI provides, in principle, a systematic method of investigating the validity of MGC models. Finally, it is noteworthy that $\mathrm{KFA}_{6 \mathrm{D}}$ is substantially larger in most of the brain than $\mathrm{KFA}_{3 \mathrm{D}}$ (see Figure 3). This suggests that the $\mathrm{KFA}_{6 \mathrm{D}}$ detects considerably more diffusion anisotropy, which presumably is mainly microscopic diffusion anisotropy not detectable with SDE MRI.

\section{CONCLUSION}

The 6D diffusion and kurtosis tensors for DP-DKI can be conveniently estimated from DDE MRI data using quadratic programming in a manner very similar to that of established post-processing methods employed for standard DKI. This includes constraints that help to 
reduce the effects of noise and imaging artifacts. Without these constraints, which typically have a significant impact on only a small fraction of the voxels, the quadratic programming algorithm reduces to a simple WLS fit of the signal model to the data. We have also introduced several rotationally invariant metrics that can be calculated from the 6D diffusion and kurtosis tensors, which may be useful in applying DP-DKI to the characterization of tissue microstructure.

\section{Acknowledgements}

This work was supported in part by a grant from the Litwin Foundation (to J. Helpern).

\section{APPENDIX A: DERIVATION OF EQUATION [9]}

The diffusion tensor has the Taylor series approximation

$$
\mathbf{D}(t) \approx \mathbf{D}\left(t_{0}\right)+\mathbf{D}^{\prime}\left(t_{0}\right)\left(t-t_{0}\right)+\frac{1}{2} \mathbf{D}^{\prime \prime}\left(t_{0}\right)\left(t-t_{0}\right)^{2}
$$

about a time $t_{0}$, where $\mathbf{D}^{\prime}(t) \equiv \partial \mathbf{D}(t) / \partial t$ and $\mathbf{D}^{\prime \prime}(t) \equiv \partial \mathbf{D}^{\prime}(t) / \partial t$. If this is applied to Equation [8], one finds

$$
\mathbf{C}(\Delta, \tau) \approx \Delta \cdot \mathbf{D}^{\prime}\left(t_{0}\right)+\frac{1}{2} \Delta\left(3 \Delta+3 \tau-2 t_{0}\right) \cdot \mathbf{D}^{\prime \prime}\left(t_{0}\right)
$$

By choosing $t_{0}=3(\Delta+\tau) / 2$, one obtains Equation [9]. This is a natural choice for $t_{0}$ since it makes the approximation insensitive to $\mathbf{D}^{\prime \prime}\left(t_{0}\right)$.

\section{APPENDIX B: EQUALITY OF $\tilde{W}_{+}^{\dagger}$, AND $\tilde{W}_{-}^{\dagger}$ FOR MGC MODELS}

From Equation [27] the difference between $\tilde{W}_{+}^{\dagger}$ and $\tilde{W}_{-}^{\dagger}$ is seen to be

$$
\tilde{W}_{+}^{\dagger}-\tilde{W}_{-}^{\dagger}=\frac{4}{5}\left(\tilde{W}_{1114}+\tilde{W}_{2225}+\tilde{W}_{3336}+\tilde{W}_{1125}+\tilde{W}_{1136}+\tilde{W}_{1224}+\tilde{W}_{1334}+\tilde{W}_{2236}+\tilde{W}_{2335}\right)
$$

Now consider an MGC model with $N$ compartments where the $n$th compartment has a water

fraction $f_{n}$ and a $6 \mathrm{D}$ diffusion tensor $\tilde{\mathbf{D}}^{(n)}$. Since we have assumed all the compartmental diffusion tensors are independent of time, the total diffusion tensor for the full model is

$$
\widetilde{\mathbf{D}}=\sum_{n=1}^{N} f_{n} \widetilde{\mathbf{D}}^{(n)}=\sum_{n=1}^{N} f_{n}\left(\begin{array}{cc}
\mathbf{D}^{(n)} & 0 \\
0 & \mathbf{D}^{(n)}
\end{array}\right),
$$


with $\mathbf{D}^{(n)}$ being the 3D diffusion tensor for the $n$th compartment. The total 6D kurtosis tensor is given by (5)

$$
\tilde{W}_{\alpha \beta \gamma \delta}=\frac{1}{\bar{D}^{2}}\left\{\left[\sum_{n=1}^{N} f_{n}\left(\tilde{D}_{\alpha \beta}^{(n)} \tilde{D}_{\gamma \delta}^{(n)}+\tilde{D}_{\alpha \gamma}^{(n)} \tilde{D}_{\beta \delta}^{(n)}+\tilde{D}_{\alpha \delta}^{(n)} \tilde{D}_{\beta \gamma}^{(n)}\right)\right]-\left(\tilde{D}_{\alpha \beta} \tilde{D}_{\gamma \delta}+\tilde{D}_{\alpha \gamma} \tilde{D}_{\beta \delta}+\tilde{D}_{\alpha \delta} \tilde{D}_{\beta \gamma}\right)\right\},
$$

where $\tilde{D}_{\alpha \beta}^{(n)}$ indicates a component of $\tilde{\mathbf{D}}^{(n)}$. From Equations [B2] and [B3], it is straightforward to verify that all the individual components of $\tilde{\mathbf{W}}$ appearing in right-hand side of Equation [B1] vanish identically, which demonstrates that $\tilde{W}_{+}^{\dagger}=\tilde{W}_{-}^{\dagger}$ for MGC models.

\section{APPENDIX C: EXPRESSION OF CUMULANT EXPANSION IN TERMS OF INDEPENDENT TENSOR COMPONENTS}

In order to implement our numerical method for calculating the $6 \mathrm{D}$ diffusion and kurtosis tensors, one needs to express the sums in Equation [34] in terms of the 12 independent components for $\tilde{\mathbf{D}}$ and 66 independent components for $\tilde{\mathbf{H}} \equiv \bar{D}^{2} \tilde{\mathbf{W}}$. Here we give explicit formulae for accomplishing this.

Let us first consider the sum

$$
\tilde{D}(\tilde{\mathbf{n}}) \equiv \sum_{\alpha, \beta=1}^{6} \tilde{n}_{\alpha} \tilde{n}_{\beta} \tilde{D}_{\alpha \beta},
$$

where the index $m$ from Equation [34] has been suppressed since it plays no essential role in the present development, which makes Equation [C1] identical to Equation [5]. As independent components for $\tilde{\mathbf{D}}$, we choose $\tilde{D}_{11}, \tilde{D}_{12}, \tilde{D}_{13}, \tilde{D}_{14}, \tilde{D}_{15}, \tilde{D}_{16}, \tilde{D}_{22}, \tilde{D}_{23}, \tilde{D}_{25}, \tilde{D}_{26}, \tilde{D}_{33}$, and $\widetilde{D}_{36}$. Any other component of $\tilde{\mathbf{D}}$ may be determined from these either by an interchange of indices or by applying the symmetry of Equation [10] or both. In terms of these components, Equation [C1] can be rewritten as

$$
\begin{aligned}
\tilde{D}(\tilde{\mathbf{n}})= & \left(\tilde{n}_{1}^{2}+\tilde{n}_{4}^{2}\right) \tilde{D}_{11}+2\left(\tilde{n}_{1} \tilde{n}_{2}+\tilde{n}_{4} \tilde{n}_{5}\right) \tilde{D}_{12}+2\left(\tilde{n}_{1} \tilde{n}_{3}+\tilde{n}_{4} \tilde{n}_{6}\right) \tilde{D}_{13}+2 \tilde{n}_{1} \tilde{n}_{4} \tilde{D}_{14} \\
& +2\left(\tilde{n}_{1} \tilde{n}_{5}+\tilde{n}_{2} \tilde{n}_{4}\right) \tilde{D}_{15}+2\left(\tilde{n}_{1} \tilde{n}_{6}+\tilde{n}_{3} \tilde{n}_{4}\right) \tilde{D}_{16}+\left(\tilde{n}_{2}^{2}+\tilde{n}_{5}^{2}\right) \tilde{D}_{22}++2\left(\tilde{n}_{2} \tilde{n}_{3}+\tilde{n}_{5} \tilde{n}_{6}\right) \tilde{D}_{23} \\
& +2 \tilde{n}_{2} \tilde{n}_{5} \tilde{D}_{25}+2\left(\tilde{n}_{2} \tilde{n}_{6}+\tilde{n}_{3} \tilde{n}_{5}\right) \tilde{D}_{26}+\left(\tilde{n}_{3}^{2}+\tilde{n}_{6}^{2}\right) \tilde{D}_{33}+2 \tilde{n}_{3} \tilde{n}_{6} \tilde{D}_{36} .
\end{aligned}
$$

The second sum in Equation [34] is

$$
\tilde{H}(\tilde{\mathbf{n}}) \equiv \sum_{\alpha, \beta, \gamma, \delta=1}^{6} \tilde{n}_{\alpha} \tilde{n}_{\beta,} \tilde{n}_{\gamma} \tilde{n}_{\delta} \tilde{H}_{\alpha \beta \gamma \delta}=\bar{D}^{2} \sum_{\alpha, \beta, \gamma, \delta=1}^{6} \tilde{n}_{\alpha} \tilde{n}_{\beta} \tilde{n}_{\gamma} \tilde{n}_{\delta} \tilde{W}_{\alpha \beta \gamma \delta},
$$


where we have again suppressed the index $m$. For the independent components of $\tilde{\mathbf{W}}$, we use $\tilde{W}_{1111}, \tilde{W}_{1112}, \tilde{W}_{1113}, \tilde{W}_{1114}, \tilde{W}_{1115}, \tilde{W}_{1116}, \tilde{W}_{1122}, \tilde{W}_{1123}, \tilde{W}_{1124}, \tilde{W}_{1125}, \tilde{W}_{1126}, \tilde{W}_{1133}, \tilde{W}_{1134}, \tilde{W}_{1135}$, $\tilde{W}_{1136}, \tilde{W}_{1144}, \tilde{W}_{1145}, \tilde{W}_{1146}, \tilde{W}_{1155}, \tilde{W}_{1156}, \tilde{W}_{1166}, \tilde{W}_{1222}, \tilde{W}_{1223}, \tilde{W}_{1224}, \tilde{W}_{1225}, \tilde{W}_{1226}, \tilde{W}_{1233}, \tilde{W}_{1234}$, $\tilde{W}_{1235}, \tilde{W}_{1236}, \tilde{W}_{1245}, \tilde{W}_{1246}, \tilde{W}_{1255}, \tilde{W}_{1256}, \tilde{W}_{1266}, \tilde{W}_{1333}, \tilde{W}_{1334}, \tilde{W}_{1335}, \tilde{W}_{1336}, \tilde{W}_{1346}, \tilde{W}_{1355}, \tilde{W}_{1356}$, $\tilde{W}_{1366}, \tilde{W}_{1555}, \tilde{W}_{1556}, \tilde{W}_{1566}, \tilde{W}_{1666}, \tilde{W}_{2222}, \tilde{W}_{2223}, \tilde{W}_{2225}, \tilde{W}_{2226}, \tilde{W}_{2233}, \tilde{W}_{2235}, \tilde{W}_{2236}, \tilde{W}_{2255}, \tilde{W}_{2256}$, $\tilde{W}_{2266}, \tilde{W}_{2333}, \tilde{W}_{2335}, \tilde{W}_{2336}, \tilde{W}_{2356}, \tilde{W}_{2366}, \tilde{W}_{2666}, \tilde{W}_{3333}, \tilde{W}_{3336}$, and $\tilde{W}_{3366}$. From these, any other component of $\tilde{\mathbf{W}}$ can be found from some combination of interchange of indices and application of the symmetry of Equation [12]. The corresponding independent components of $\tilde{\mathbf{H}}$ are simply $\bar{D}^{2}$ times the independent components for $\tilde{\mathbf{W}}$. Using the 66 independent components of $\tilde{\mathbf{W}}$, Equation [C3] takes the form

$$
\begin{aligned}
& \tilde{H}(\tilde{\mathbf{n}})=\bar{D}^{2}\left[\left(\tilde{n}_{1}^{4}+\tilde{n}_{4}^{4}\right) \tilde{W}_{1111}+4\left(\tilde{n}_{1}^{3} \tilde{n}_{2}+\tilde{n}_{4}^{3} \tilde{n}_{5}\right) \tilde{W}_{1112}+4\left(\tilde{n}_{1}^{3} \tilde{n}_{3}+\tilde{n}_{4}^{3} \tilde{n}_{6}\right) \tilde{W}_{1113}\right. \\
& +4\left(\tilde{n}_{1}^{3} \tilde{n}_{4}+\tilde{n}_{1} \tilde{n}_{4}^{3}\right) \tilde{W}_{1114}+4\left(\tilde{n}_{1}^{3} \tilde{n}_{5}+\tilde{n}_{2} \tilde{n}_{4}^{3}\right) \tilde{W}_{1115}+4\left(\tilde{n}_{1}^{3} \tilde{n}_{6}+\tilde{n}_{3} \tilde{n}_{4}^{3}\right) \tilde{W}_{1116} \\
& +6\left(\tilde{n}_{1}^{2} \tilde{n}_{2}^{2}+\tilde{n}_{4}^{2} \tilde{n}_{5}^{2}\right) \tilde{W}_{1122}+12\left(\tilde{n}_{1}^{2} \widetilde{n}_{2} \widetilde{n}_{3}+\tilde{n}_{4}^{2} \widetilde{n}_{5} \tilde{n}_{6}\right) \tilde{W}_{1123}+12\left(\tilde{n}_{1}^{2} \widetilde{n}_{2} \tilde{n}_{4}+\tilde{n}_{1} \tilde{n}_{4}^{2} \tilde{n}_{5}\right) \tilde{W}_{1124} \\
& +12\left(\tilde{n}_{1}^{2} \tilde{n}_{2} \tilde{n}_{5}+\tilde{n}_{2} \tilde{n}_{4}^{2} \tilde{n}_{5}\right) \tilde{W}_{1125}+12\left(\tilde{n}_{1}^{2} \tilde{n}_{2} \tilde{n}_{6}+\tilde{n}_{3} \tilde{n}_{4}^{2} \tilde{n}_{5}\right) \tilde{W}_{1126}+6\left(\tilde{n}_{1}^{2} \tilde{n}_{3}^{2}+\tilde{n}_{4}^{2} \tilde{n}_{6}^{2}\right) \tilde{W}_{1133} \\
& +12\left(\tilde{n}_{1}^{2} \tilde{n}_{3} \tilde{n}_{4}+\tilde{n}_{1} \tilde{n}_{4}^{2} \tilde{n}_{6}\right) \tilde{W}_{1134}+12\left(\tilde{n}_{1}^{2} \tilde{n}_{3} \tilde{n}_{5}+\tilde{n}_{2} \tilde{n}_{4}^{2} \tilde{n}_{6}\right) \tilde{W}_{1135}+12\left(\tilde{n}_{1}^{2} \tilde{n}_{3} \tilde{n}_{6}+\tilde{n}_{3} \tilde{n}_{4}^{2} \tilde{n}_{6}\right) \tilde{W}_{1136} \\
& +6 \tilde{n}_{1}^{2} \tilde{n}_{4}^{2} \tilde{W}_{1144}+12\left(\tilde{n}_{1}^{2} \tilde{n}_{4} \tilde{n}_{5}+\tilde{n}_{1} \tilde{n}_{2} \tilde{n}_{4}^{2}\right) \tilde{W}_{1145}+12\left(\tilde{n}_{1}^{2} \tilde{n}_{4} \tilde{n}_{6}+\tilde{n}_{1} \tilde{n}_{3} \tilde{n}_{4}^{2}\right) \tilde{W}_{1146} \\
& +6\left(\tilde{n}_{1}^{2} \tilde{n}_{5}^{2}+\tilde{n}_{2}^{2} \tilde{n}_{4}^{2}\right) \tilde{W}_{1155}+12\left(\tilde{n}_{1}^{2} \tilde{n}_{5} \tilde{n}_{6}+\tilde{n}_{2} \tilde{n}_{3} \tilde{n}_{4}^{2}\right) \tilde{W}_{1156}+6\left(\tilde{n}_{1}^{2} \tilde{n}_{6}^{2}+\tilde{n}_{3}^{2} \tilde{n}_{4}^{2}\right) \tilde{W}_{1166} \\
& +4\left(\tilde{n}_{1} \tilde{n}_{2}^{3}+\tilde{n}_{4} \tilde{n}_{5}^{3}\right) \tilde{W}_{1222}+12\left(\tilde{n}_{1} \tilde{n}_{2}^{2} \widetilde{n}_{3}+\tilde{n}_{4} \tilde{n}_{5}^{2} \tilde{n}_{6}\right) \tilde{W}_{1223}+12\left(\tilde{n}_{1} \tilde{n}_{2}^{2} \tilde{n}_{4}+\tilde{n}_{1} \tilde{n}_{4} \tilde{n}_{5}^{2}\right) \tilde{W}_{1224} \\
& +12\left(\tilde{n}_{1} \tilde{n}_{2}^{2} \tilde{n}_{5}+\tilde{n}_{2} \tilde{n}_{4} \tilde{n}_{5}^{2}\right) \tilde{W}_{1225}+12\left(\tilde{n}_{1} \tilde{n}_{2}^{2} \tilde{n}_{6}+\tilde{n}_{3} \tilde{n}_{4} \tilde{n}_{5}^{2}\right) \tilde{W}_{1226}+12\left(\tilde{n}_{1} \tilde{n}_{2} \tilde{n}_{3}^{2}+\tilde{n}_{4} \tilde{n}_{5} \tilde{n}_{6}^{2}\right) \tilde{W}_{1233} \\
& +24\left(\tilde{n}_{1} \tilde{n}_{2} \tilde{n}_{3} \tilde{n}_{4}+\tilde{n}_{1} \tilde{n}_{4} \tilde{n}_{5} \tilde{n}_{6}\right) \tilde{W}_{1234}+24\left(\tilde{n}_{1} \tilde{n}_{2} \tilde{n}_{3} \tilde{n}_{5}+\tilde{n}_{2} \tilde{n}_{4} \tilde{n}_{5} \tilde{n}_{6}\right) \tilde{W}_{1235}+24\left(\tilde{n}_{1} \tilde{n}_{2} \tilde{n}_{3} \tilde{n}_{6}+\tilde{n}_{3} \tilde{n}_{4} \tilde{n}_{5} \tilde{n}_{6}\right) \tilde{W}_{1236}
\end{aligned}
$$

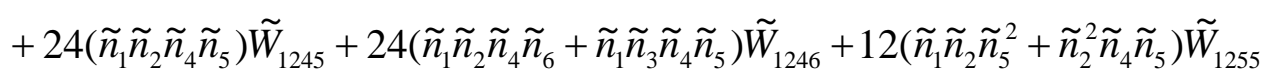

$$
\begin{aligned}
& +24\left(\tilde{n}_{1} \tilde{n}_{2} \tilde{n}_{5} \tilde{n}_{6}+\tilde{n}_{2} \widetilde{n}_{3} \widetilde{n}_{4} \tilde{n}_{5}\right) \tilde{W}_{1256}+12\left(\tilde{n}_{1} \tilde{n}_{2} \tilde{n}_{6}^{2}+\tilde{n}_{3}^{2} \widetilde{n}_{4} \tilde{n}_{5}\right) \tilde{W}_{1266}+4\left(\tilde{n}_{1} \tilde{n}_{3}^{3}+\tilde{n}_{4} \tilde{n}_{6}^{3}\right) \tilde{W}_{1333} \\
& +12\left(\tilde{n}_{1} \tilde{n}_{3}^{2} \tilde{n}_{4}+\tilde{n}_{1} \tilde{n}_{4} \tilde{n}_{6}^{2}\right) \tilde{W}_{1334}+12\left(\tilde{n}_{1} \tilde{n}_{3}^{2} \tilde{n}_{5}+\tilde{n}_{2} \tilde{n}_{4} \tilde{n}_{6}^{2}\right) \tilde{W}_{1335}+12\left(\tilde{n}_{1} \tilde{n}_{3}^{2} \tilde{n}_{6}+\tilde{n}_{3} \tilde{n}_{4} \tilde{n}_{6}^{2}\right) \tilde{W}_{1336} \\
& +24\left(\tilde{n}_{1} \tilde{n}_{3} \tilde{n}_{4} \tilde{n}_{6}\right) \tilde{W}_{1346}+12\left(\tilde{n}_{1} \tilde{n}_{3} \tilde{n}_{5}^{2}+\tilde{n}_{2}^{2} \tilde{n}_{4} \tilde{n}_{6}\right) \tilde{W}_{1355}+24\left(\tilde{n}_{1} \tilde{n}_{3} \tilde{n}_{5} \tilde{n}_{6}+\tilde{n}_{2} \tilde{n}_{3} \tilde{n}_{4} \tilde{n}_{6}\right) \tilde{W}_{1356} \\
& +12\left(\tilde{n}_{1} \tilde{n}_{3} \tilde{n}_{6}^{2}+\tilde{n}_{3}^{2} \tilde{n}_{4} \tilde{n}_{6}\right) \tilde{W}_{1366}+4\left(\tilde{n}_{1} \tilde{n}_{5}^{3}+\tilde{n}_{2}^{3} \tilde{n}_{4}\right) \tilde{W}_{1555}+12\left(\tilde{n}_{1} \tilde{n}_{5}^{2} \tilde{n}_{6}+\tilde{n}_{2}^{2} \tilde{n}_{3} \tilde{n}_{4}\right) \tilde{W}_{1556} \\
& +12\left(\tilde{n}_{1} \tilde{n}_{5} \tilde{n}_{6}^{2}+\tilde{n}_{2} \tilde{n}_{3}^{2} \tilde{n}_{4}\right) \tilde{W}_{1566}+4\left(\tilde{n}_{1} \tilde{n}_{6}^{3}+\tilde{n}_{3}^{3} \tilde{n}_{4}\right) \tilde{W}_{1666}+\left(\tilde{n}_{2}^{4}+\tilde{n}_{5}^{4}\right) \tilde{W}_{2222}
\end{aligned}
$$




$$
\begin{aligned}
& +4\left(\tilde{n}_{2}^{3} \tilde{n}_{3}+\tilde{n}_{5}^{3} \tilde{n}_{6}\right) \tilde{W}_{2223}+4\left(\tilde{n}_{2}^{3} \tilde{n}_{5}+\tilde{n}_{2} \tilde{n}_{5}^{3}\right) \tilde{W}_{2225}+4\left(\tilde{n}_{2}^{3} \tilde{n}_{5}+\tilde{n}_{2} \tilde{n}_{5}^{3}\right) \tilde{W}_{2225} \\
& +4\left(\tilde{n}_{2}^{3} \tilde{n}_{6}+\tilde{n}_{3} \tilde{n}_{5}^{3}\right) \tilde{W}_{2226}+6\left(\tilde{n}_{2}^{2} \tilde{n}_{3}^{2}+\tilde{n}_{5}^{2} \tilde{n}_{6}^{2}\right) \tilde{W}_{2233}+12\left(\tilde{n}_{2}^{2} \tilde{n}_{3} \tilde{n}_{5}+\tilde{n}_{2} \tilde{n}_{5}^{2} \tilde{n}_{6}\right) \tilde{W}_{2235} \\
& +12\left(\tilde{n}_{2}^{2} \widetilde{n}_{3} \tilde{n}_{6}+\tilde{n}_{3} \tilde{n}_{5}^{2} \widetilde{n}_{6}\right) \tilde{W}_{2236}+6 \tilde{n}_{2}^{2} \widetilde{n}_{5}^{2} \tilde{W}_{2255}+12\left(\tilde{n}_{2}^{2} \widetilde{n}_{5} \tilde{n}_{6}+\tilde{n}_{2} \tilde{n}_{3} \tilde{n}_{5}^{2}\right) \tilde{W}_{2256} \\
& +6\left(\tilde{n}_{2}^{2} \tilde{n}_{6}^{2}+\tilde{n}_{3}^{2} \tilde{n}_{5}^{2}\right) \tilde{W}_{2266}+4\left(\tilde{n}_{2} \tilde{n}_{3}^{3}+\tilde{n}_{5} \tilde{n}_{6}^{3}\right) \tilde{W}_{2333}+12\left(\tilde{n}_{2} \tilde{n}_{3}^{2} \tilde{n}_{5}+\tilde{n}_{2} \tilde{n}_{5} \tilde{n}_{6}^{2}\right) \tilde{W}_{2335} \\
& +12\left(\tilde{n}_{2} \tilde{n}_{3}^{2} \tilde{n}_{6}+\tilde{n}_{3} \tilde{n}_{5} \tilde{n}_{6}^{2}\right) \tilde{W}_{2336}+24\left(\tilde{n}_{2} \tilde{n}_{3} \tilde{n}_{5} \tilde{n}_{6}\right) W_{2356}+12\left(\tilde{n}_{2} \tilde{n}_{3} \tilde{n}_{6}^{2}+\tilde{n}_{3}^{2} \tilde{n}_{5} \tilde{n}_{6}\right) \tilde{W}_{2366} \\
& \left.+4\left(\tilde{n}_{2} \tilde{n}_{6}^{3}+\tilde{n}_{3}^{3} \tilde{n}_{5}\right) \tilde{W}_{2666}+\left(\tilde{n}_{3}^{4}+\tilde{n}_{6}^{4}\right) \tilde{W}_{3333}+4\left(\tilde{n}_{3}^{3} \tilde{n}_{6}+\tilde{n}_{3} \tilde{n}_{6}^{3}\right) \tilde{W}_{3666}+6 \tilde{n}_{3}^{2} \tilde{n}_{6}^{2} \tilde{W}_{3366}\right]
\end{aligned}
$$

\section{APPENDIX D: CONSTRUCTING MATRICES A AND G}

By combining Equations [C1], [34], and [C3], one can show that

$$
Y_{m}=-\tilde{b}_{m} \tilde{D}\left(\tilde{\mathbf{n}}_{m}\right)+\frac{\tilde{b}_{m}^{2}}{6} \tilde{H}\left(\tilde{\mathbf{n}}_{m}\right), \quad \text { for } m=1,2, \ldots, M
$$

Since $\mathbf{Y}=\mathbf{A X}$ and $\mathbf{X}$ is a vector composed of the $12+66=78$ independent components for $\tilde{\mathbf{D}}$ and $\tilde{\mathbf{H}}$, the components for A may be easily read off by comparing Equations [C2] and [C4] with [D1]. This is illustrated by the following examples:

$$
\begin{aligned}
& A_{m, 1}=-\tilde{b}_{m}\left(\tilde{n}_{1, m}^{2}+\tilde{n}_{4, m}^{2}\right), \\
& A_{m, 6}=-2 \tilde{b}_{m}\left(\tilde{n}_{1, m} \tilde{n}_{6, m}+\tilde{n}_{3, m} \tilde{n}_{4, m}\right), \\
& A_{m, 12}=-2 \tilde{b}_{m} \tilde{n}_{3, m} \tilde{n}_{6, m}, \\
& A_{m, 13}=\frac{\tilde{b}_{m}^{2}}{6}\left(\tilde{n}_{1, m}^{4}+\tilde{n}_{4, m}^{4}\right), \\
& A_{m, 21}=2 \tilde{b}_{m}^{2}\left(\tilde{n}_{1, m}^{2} \tilde{n}_{2, m} \tilde{n}_{4, m}+\tilde{n}_{1, m} \tilde{n}_{4, m}^{2} \tilde{n}_{5, m}\right), \\
& A_{m, 78}=\tilde{b}_{m}^{2} \tilde{n}_{3, m}^{2} \tilde{n}_{6, m}^{2} .
\end{aligned}
$$

The constraints of Equations [40] and [41] may be rewritten as

$$
-\tilde{H}\left(\tilde{\mathbf{n}}_{m}\right)<0
$$

and

$$
\tilde{b}_{\max } \tilde{H}\left(\tilde{\mathbf{n}}_{m}\right)-3 \tilde{D}\left(\tilde{\mathbf{n}}_{m}\right)<0,
$$

for all $m=1,2, \ldots, M$. The matrix $\mathbf{G}$ is defined so that Equations [D3] and [D4] are equivalent to the system $\mathbf{G X}<0$. The $2 M \times 78$ components of $\mathbf{G}$ can be found by comparing Equations [C2] and [C4] with [D3] and [D4]. For instance, we have 


$$
\begin{aligned}
& G_{m, 1}=0, \\
& G_{m, 6}=0, \\
& G_{m, 12}=0, \\
& G_{m, 13}=-\left(\tilde{n}_{1, m}^{4}+\tilde{n}_{4, m}^{4}\right), \\
& G_{m, 21}=-12\left(\tilde{n}_{1, m}^{2} \tilde{n}_{2, m} \tilde{n}_{4, m}+\tilde{n}_{1, m} \tilde{n}_{4, m}^{2} \tilde{n}_{5, m}\right), \\
& G_{m, 78}=-6 \tilde{n}_{3, m}^{2} \tilde{n}_{6, m}^{2},
\end{aligned}
$$

and

$$
\begin{aligned}
& G_{m+M, 1}=-3\left(\tilde{n}_{1, m}^{2}+\tilde{n}_{4, m}^{2}\right), \\
& G_{m+M, 6}=-6\left(\tilde{n}_{1, m} \tilde{n}_{6, m}+\tilde{n}_{3, m} \tilde{n}_{4, m}\right), \\
& G_{m+M, 12}=-6 \tilde{n}_{3, m} \tilde{n}_{6, m}, \\
& G_{m+M, 13}=\tilde{b}_{\max }\left(\tilde{n}_{1, m}^{4}+\tilde{n}_{4, m}^{4}\right), \\
& G_{m+M, 21}=12 \tilde{b}_{\max }\left(\tilde{n}_{1, m}^{2} \tilde{n}_{2, m} \tilde{n}_{4, m}+\tilde{n}_{1, m} \tilde{n}_{4, m}^{2} \tilde{r}_{5, m}\right), \\
& G_{m+M, 78}=6 \tilde{b}_{\max } \tilde{n}_{3, m}^{2} \tilde{n}_{6, m}^{2} .
\end{aligned}
$$

for $m=1,2, \ldots, M$.

\section{APPENDIX E: 6D DIFFUSION ENCODING DIRECTIONS}

Table 1. The 80 6D diffusion encoding directions utilized for the data acquisition and analysis.

\begin{tabular}{|c|c|c|c|}
\hline$m$ & $\tilde{\mathbf{n}}_{m}$ & $m$ & $\tilde{\mathbf{n}}_{m}$ \\
\hline 1 & $(1,0,0,0,0,0)$ & 41 & $(0.3456,0.2255,0.3225,-0.7599,-0.1278,0.3630)$ \\
\hline 2 & $(0,1,0,0,0,0)$ & 42 & $(0.2730,0.0911,-0.7255,-0.3081,0.5235,-0.1469)$ \\
\hline 3 & $(0,0,1,0,0,0)$ & 43 & $(-0.2738,-0.5635,-0.1612,0.6311,-0.2389,-0.3551)$ \\
\hline 4 & $(1,1,0,0,0,0) / \sqrt{2}$ & 44 & $(-0.6144,0.1429,0.1611,0.1019,0.0229,0.7517)$ \\
\hline 5 & $(1,-1,0,0,0,0) / \sqrt{2}$ & 45 & $(0.0567,-0.3176,0.1117,-0.3639,0.5460,-0.6728)$ \\
\hline 6 & $(1,0,1,0,0,0) / \sqrt{2}$ & 46 & $(0.6549,-0.3068,0.4709,-0.3602,0.1134,0.3352)$ \\
\hline 7 & $(1,0,-1,0,0,0) / \sqrt{2}$ & 47 & $(-0.6180,-0.3746,0.1895,-0.3830,0.2089,0.5013)$ \\
\hline 8 & $(0,1,1,0,0,0) / \sqrt{2}$ & 48 & $(0.1778,0.5368,0.1873,0.5629,0.4084,0.4017)$ \\
\hline 9 & $(0,1,-1,0,0,0) / \sqrt{2}$ & 49 & $(-0.4994,-0.1890,-0.4568,-0.5835,0.0023,0.4069)$ \\
\hline 10 & $(1,0,0,0,1,0) / \sqrt{2}$ & 50 & $(0.1428,-0.0224,0.4515,0.6281,0.5415,-0.2956)$ \\
\hline
\end{tabular}




\begin{tabular}{|c|c|c|c|}
\hline 11 & $(1,0,0,0,-1,0) / \sqrt{2}$ & 51 & $(-0.4176,-0.4481,0.0320,0.5131,0.5990,0.0391)$ \\
\hline 12 & $(1,0,0,0,0,1) / \sqrt{2}$ & 52 & $(0.0778,-0.7125,0.2687,-0.5743,-0.1641,0.2389)$ \\
\hline 13 & $(1,0,0,0,0,-1) / \sqrt{2}$ & 53 & $(0.2799,-0.5163,-0.3685,0.6873,0.1105,-0.1856)$ \\
\hline 14 & $(0,1,0,0,0,1) / \sqrt{2}$ & 54 & $(-0.5564,-0.01271,0.2968,-0.7376,0.2339,0.0561)$ \\
\hline 15 & $(0,1,0,0,0,-1) / \sqrt{2}$ & 55 & $(0.3422,0.3006,0.7451,-0.3400,-0.02450 .3477)$ \\
\hline 16 & $(1,0,0,1,0,0) / \sqrt{2}$ & 56 & $(-0.7780,0.3670,0.0501,0.4551,0.2235,-0.0154)$ \\
\hline 17 & $(1,0,0,-1,0,0) / \sqrt{2}$ & 57 & $(-0.0203,0.1160,-0.4572,-0.8122,-0.0584,-0.3375)$ \\
\hline 18 & $(0,1,0,0,1,0) / \sqrt{2}$ & 58 & $(0.4422,-0.4742,-0.2065,-0.4800,0.5527,0.0295)$ \\
\hline 19 & $(0,1,0,0,-1,0) / \sqrt{2}$ & 59 & $(-0.4492,-0.0022,-0.6925,-0.4220,-0.2992,-0.2253)$ \\
\hline 20 & $(0,0,1,0,0,-1) / \sqrt{2}$ & 60 & $(0.2942,0.3001,-0.1773,-0.1848,-0.8671,0.0753)$ \\
\hline 21 & $(0,0,1,0,0,-1) / \sqrt{2}$ & 61 & $(0.1543,-0.7366,-0.2666,0.4773,-0.1862,-0.3160)$ \\
\hline 22 & $(-0.2811,-0.4601,-0.70249,0.2928,-0.1600)$ & 62 & $(0.5569,-0.2630,0.6358,-0.1686,0.4302,0.0519)$ \\
\hline 23 & $(0.3850,0.5550,-0.1133,-0.1068,0.4731,-0.5436)$ & 63 & $(-0.0179,0.7224,0.1612,0.1725,0.6386,0.1186)$ \\
\hline 24 & $(-0.2699,0.4698,-0.4116,-0.3017,0.5460,0.3841)$ & 64 & $(-0.3041,-0.2436,-0.3301,0.2978,-0.7434,-0.3125)$ \\
\hline 25 & $(0.6230,0.5213,-0.2129,0.2202,-0.3474,0.3540)$ & 65 & $(-0.0715,0.6053,-0.1692,0.0232,-0.0818,-0.7697)$ \\
\hline 26 & $(0.5522,0.4189,0.4199,-0.1749,0.4852,0.2777)$ & 66 & $(-0.0798,-0.3675,0.1451,0.5093,0.6519,-0.3910)$ \\
\hline 27 & $(0.5683,-0.2285,0.6934,-0.0356,-0.0631,0.3723)$ & 67 & $(-0.3433,-0.0263,-0.5302,0.5692,-0.5025,0.1538)$ \\
\hline 28 & $(-0.4323,-0.2239,-0.2699,-0.7015,0.4432,0.0378)$ & 68 & $(-0.3292,0.3152,0.5382,-0.5180,0.4413,0.1983)$ \\
\hline 29 & $(-0.4989,0.0430,-0.7226,0.2070,0.3797,-0.1997)$ & 69 & $(-0.6441,0.1404,0.4375,0.4965,-0.0278,-0.3557)$ \\
\hline 30 & $(-0.1633,-0.7634,-0.3950,-0.4495,-0.1783,-0.0237)$ & 70 & $(-0.4113,0.0377,-0.8172,0.1923,0.3528,0.0051)$ \\
\hline 31 & $(0.1484,-0.3996,-0.7159,-0.4634,-0.1962,0.2288)$ & 71 & $(-0.6476,-0.1106,0.0324,0.1381,-0.4521,-0.5862)$ \\
\hline 32 & $(0.0334,0.3940,-0.4010,-0.4920,-0.2704,0.6062)$ & 72 & $(-0.2679,-0.0698,0.6735,-0.3179,0.4054,0.4518)$ \\
\hline 33 & $(-0.5925,-0.2337,-0.3887,-0.1743,0.6398,0.0570)$ & 73 & $(0.1068,-0.2321,0.5544,-0.2748,0.5051,-0.5445)$ \\
\hline 34 & $(0.3312,-0.1699,-0.8752,0.1653,-0.1146,-0.2342)$ & 74 & $(-0.3387,0.3623,0.0564,-0.7209,0.30148,0.3743)$ \\
\hline 35 & $(0.1965,0.2373,0.1676,-0.4726,-0.6522,0.4776)$ & 75 & $(0.5899,-0.3189,-0.7207,0.0120,-0.1746,-0.0151)$ \\
\hline 36 & $(-0.0724,-0.8867,-0.2288,-0.0695,0.3750,-0.1023)$ & 76 & $(0.1763,-0.0660,-0.7675,-0.2409,0.2165,0.5201)$ \\
\hline 37 & $(0.3251,0.5947,0.4815,0.0674,0.3835,-0.3961)$ & 77 & $(-0.0555,0.4035,-0.0747,-0.1348,0.4544,-0.7769)$ \\
\hline
\end{tabular}




\begin{tabular}{|c|c|c|c|}
\hline 38 & $(0.0885,-0.3858,0.3950,0.5256,0.1682,-0.6184)$ & 78 & $(-0.2290,0.4984,0.3256,-0.0277,-0.7614,0.1116)$ \\
\hline 39 & $(0.2134,0.2114,-0.0669,-0.4148,0.6893,0.5078)$ & 79 & $(-0.0531,-0.8513,0.2401,-0.4233,0.1584,0.1017)$ \\
\hline 40 & $(-0.2179,-0.5804,-0.6440,0.3290,0.1408,-0.2695)$ & 80 & $(0.5263,-0.1589,0.6515,0.0085,0.5207,-0.0430)$ \\
\hline
\end{tabular}




\section{REFERENCES}

1. Shemesh N, Ozarslan E, Komlosh ME, Basser PJ, Cohen Y. From single-pulsed field gradient to double-pulsed field gradient MR: gleaning new microstructural information and developing new forms of contrast in MRI. NMR Biomed 2010;23(7):757-780.

2. Shemesh N, Jespersen SN, Alexander DC, Cohen Y, Drobnjak I, Dyrby TB, Finsterbusch J, Koch MA, Kuder T, Laun F, Lawrenz M, Lundell H, Mitra PP, Nilsson M, Özarslan E, Topgaard D, Westin CF. Conventions and nomenclature for double diffusion encoding NMR and MRI. Magn Reson Med 2016;75(1):82-87.

3. Lawrenz M, Koch MA, Finsterbusch J. A tensor model and measures of microscopic anisotropy for double-wave-vector diffusion-weighting experiments with long mixing times. J Magn Reson 2010;202(1):43-56.

4. Jespersen SN, Lundell H, Sønderby CK, Dyrby TB. Orientationally invariant metrics of apparent compartment eccentricity from double pulsed field gradient diffusion experiments. NMR Biomed 2013;26(12):1647-1662.

5. Jensen JH, Hui ES, Helpern JA. Double-pulsed diffusional kurtosis imaging. NMR Biomed 2014;27(4):363-370.

6. Lawrenz M, Finsterbusch J. Mapping measures of microscopic diffusion anisotropy in human brain white matter in vivo with double-wave-vector diffusion-weighted imaging. Magn Reson Med 2015;73(2):773-783.

7. Hui ES, Jensen JH. Double-pulsed diffusional kurtosis imaging for the in vivo assessment of human brain microstructure. Neuroimage 2015;120:371-381.

8. Komlosh ME, Özarslan E, Lizak MJ, Horkay F, Schram V, Shemesh N, Cohen Y, Basser PJ. Pore diameter mapping using double pulsed-field gradient MRI and its validation using a novel glass capillary array phantom. J Magn Reson 2011;208(1):128-135.

9. Shemesh N, Cohen Y. Microscopic and compartment shape anisotropies in gray and white matter revealed by angular bipolar double-PFG MR. Magn Reson Med. 2011 May;65(5):1216-1227.

10. Koch MA, Finsterbusch J. Towards compartment size estimation in vivo based on double wave vector diffusion weighting. NMR Biomed 2011;24(10):1422-1432.

11. Lawrenz M, Brassen S, Finsterbusch J. Microscopic diffusion anisotropy in the human brain: Age-related changes. Neuroimage 2016;141:313-325. 
12. Uğurbil K, Xu J, Auerbach EJ, Moeller S, Vu AT, Duarte-Carvajalino JM, Lenglet C, Wu X, Schmitter S, Van de Moortele PF, Strupp J, Sapiro G, De Martino F, Wang D, Harel N, Garwood M, Chen L, Feinberg DA, Smith SM, Miller KL, Sotiropoulos SN, Jbabdi S, Andersson JL, Behrens TE, Glasser MF, Van Essen DC, Yacoub E. Pushing spatial and temporal resolution for functional and diffusion MRI in the Human Connectome Project. Neuroimage. 2013;80:80-104.

13. Basser PJ. Relationships between diffusion tensor and q-space MRI. Magn Reson Med 2002;47(2):392-397.

14. Jensen JH, Helpern JA. MRI quantification of non-Gaussian water diffusion by kurtosis analysis. NMR Biomed 2010;23(7):698-710.

15. Kiselev VG. The cumulant expansion: an overarching mathematical framework for understanding diffusion NMR. In: Jones DK, editor. Diffusion MRI: theory, methods, and applications. New York: Oxford University Press; 2011. pp. 152-168.

16. Jespersen SN, Buhl N. The displacement correlation tensor: microstructure, ensemble anisotropy and curving fibers. J Magn Reson 2011;208:34-43.

17. Jespersen SN. Equivalence of double and single wave vector diffusion contrast at low diffusion weighting. NMR Biomed 2012;25(6):813-818.

18. Jensen JH. Stejskal's formula for multiple-pulsed diffusion MRI. Magn Reson Imaging 2015;33(9):1182-1186.

19. Hansen B, Lund TE, Sangill R, Jespersen SN. Experimentally and computationally fast method for estimation of a mean kurtosis. Magn Reson Med 2013;69(6):1754-1760.

20. Tabesh A, Jensen JH, Ardekani BA, Helpern JA. Estimation of tensors and tensorderived measures in diffusional kurtosis imaging. Magn Reson Med 2011;65(3):823-836.

21. Veraart J, Rajan J, Peeters RR, Leemans A, Sunaert S, Sijbers J. Comprehensive framework for accurate diffusion MRI parameter estimation. Magn Reson Med 2013;70(4):972-984.

22. Boggs PT, Tolle JW. Sequential quadratic programming. Acta Numerica 1995;4:1-51.

23. Jensen JH. Sufficiency of diffusion tensor in characterizing the diffusion MRI signal to leading order in diffusion weighting. NMR Biomed 2014;27(8):1005-1007.

24. Glenn GR, Helpern JA, Tabesh A, Jensen JH. Quantitative assessment of diffusional kurtosis anisotropy. NMR Biomed 2015;28(4):448-459. 
25. Available at: http://www.nitrc.org/projects/dke

26. Veraart J, Poot DH, Van Hecke W, Blockx I, Van der Linden A, Verhoye M, Sijbers J. More accurate estimation of diffusion tensor parameters using diffusion kurtosis imaging. Magn Reson Med 2011;65(1):138-145.

27. Jensen JH, Helpern JA, Ramani A, Lu H, Kaczynski K. Diffusional kurtosis imaging: the quantification of non-Gaussian water diffusion by means of magnetic resonance imaging. Magn Reson Med 2005;53(6):1432-1440.

28. Lazar M, Jensen JH, Xuan L, Helpern JA. Estimation of the orientation distribution function from diffusional kurtosis imaging. Magn Reson Med 2008;60(4):774-781.

29. Wolfe P. The simplex method for quadratic programming. Econometrica 1959;27(3):382- R2,C16 398.

30. Skare S, Hedehus M, Moseley ME, Li TQ. Condition number as a measure of noise performance of diffusion tensor data acquisition schemes with MRI. J Magn Reson 2000;147(2):340-352.

31. Lasič S, Szczepankiewicz F, Eriksson S, Nilsson M, Topgaard D. Microanisotropy imaging: quantification of microscopic diffusion anisotropy and orientational order parameter by diffusion MRI with magic-angle spinning of the q-vector. Frontiers in Physics 2014;2:11.

32. Szczepankiewicz F, Lasič S, van Westen D, Sundgren PC, Englund E, Westin CF, Ståhlberg F, Lätt J, Topgaard D, Nilsson M. Quantification of microscopic diffusion anisotropy disentangles effects of orientation dispersion from microstructure: applications in healthy volunteers and in brain tumors. Neuroimage 2015;104:241-252.

33. Paulsen JL, Özarslan E, Komlosh ME, Basser PJ, Song YQ. Detecting compartmental non-Gaussian diffusion with symmetrized double-PFG MRI. NMR Biomed 2015;28(11):1550-1556.

34. Szczepankiewicz F, van Westen D, Englund E, Westin CF, Ståhlberg F, Lätt J, Sundgren PC, Nilsson M. The link between diffusion MRI and tumor heterogeneity: Mapping cell eccentricity and density by diffusional variance decomposition (DIVIDE). Neuroimage 2016. DOI: 10.1016/j.neuroimage.2016.07.038. 
35. Fieremans E, Burcaw LM, Lee HH, Lemberskiy G, Veraart J, Novikov DS. In vivo observation and biophysical interpretation of time-dependent diffusion in human white matter. Neuroimage 2016;129:414-427.

36. Panagiotaki E, Schneider T, Siow B, Hall MG, Lythgoe MF, Alexander DC. Compartment models of the diffusion MR signal in brain white matter: a taxonomy and comparison. Neuroimage 2012;59(3):2241-2254. 


\section{FIGURE CAPTIONS:}

1. Representative axial maps for two subjects showing the diffusivity metrics, $\bar{D}, \bar{C}, \tilde{D}_{+}^{\dagger}$, and $\tilde{D}_{-}^{\dagger}$, as determined with DP-DKI using the constrained WLS method defined by Equations [42] and [43]. Within brain tissue, the magnitude of $\bar{C}$ is seen to be small, indicating a weak dependence of the diffusivity on diffusion time over the time scale probed by our DDE MRI sequence. Somewhat elevated $\bar{C}$ values are observed in some regions containing cerebrospinal fluid, which may be due to time-dependent changes in flow. The calibration bars for $\bar{D}, \bar{C}, \tilde{D}_{+}^{\dagger}$, and $\tilde{D}_{-}^{\dagger}$ are in units of $\mu \mathrm{m}^{2} / \mathrm{ms}$.

2. Representative maps estimated with DP-DKI showing four independent rotational variants, $\bar{W}, \widetilde{\bar{W}}, \tilde{W}_{+}^{\dagger}$, and $\tilde{W}_{-}^{\dagger}$, that are linear in the kurtosis tensor. The parameter $\bar{W}$ can also be measured with conventional DKI, but the other three invariants require a DDE MRI method such as DP-DKI. Note the distinct contrast for each metric, reflecting the unique physical information that they quantify. Also shown is the difference $\delta \bar{W}=\widetilde{\bar{W}}-\widetilde{\bar{W}}$, which has been proposed an index of microscopic anisotropy $(5,7)$. All R2,C35 calibration bars are dimensionless.

3. Maps of the fractional anisotropies for the $3 \mathrm{D}$ diffusion tensor ( $\left.F A_{3 \mathrm{D}}\right), 6 \mathrm{D}$ diffusion tensor $\left(\mathrm{FA}_{6 \mathrm{D}}\right)$, 3D kurtosis tensor $\left(\mathrm{KFA}_{3 \mathrm{D}}\right)$, and 6D kurtosis tensor ( $\left.\mathrm{KFA}_{6 \mathrm{D}}\right)$, along with the microscopic fractional anisotropy $(\mu \mathrm{FA})$. Note that while the $\mathrm{FA}_{3 \mathrm{D}}$ and $\mathrm{FA}_{6 \mathrm{D}}$ maps are nearly identical the $\mathrm{KFA}_{3 \mathrm{D}}$ and $\mathrm{KFA}_{6 \mathrm{D}}$ are substantially different. Of these five rotational invariants, only $\mathrm{KFA}_{6 \mathrm{D}}$ and $\mu \mathrm{FA}$ are impossible to estimate with SDE methods, and so they provide the most novel information. All calibration bars are dimensionless.

4. Comparison of the four linear kurtosis metrics of $\bar{W}, \stackrel{\widetilde{W}}{,} \tilde{W}_{+}^{\dagger}$, and $\tilde{W}_{-}^{\dagger}$, as obtained from minimizing the cost function of Equation [42] with and without applying the constraints 
of Equations [43]. Several prominent hypointense regions are apparent for the unconstrained calculations, which are mitigated or eliminated when the constraints are imposed.

5. A comparison of the measured and fitted values for $Y_{m}$ as defined by Equation [33] for ROIs (red squares) in the (a) corpus callosum, (b) prefrontal white matter, and (c) thalamus from Subject 1 . The plots show their corresponding measured (left-hand side of Equation [34]) and modeled (right-hand side of Equation [34]) data points along each gradient direction $\left(\tilde{\mathbf{n}}_{m}\right)$ as a function of diffusion weighting $(\tilde{b})$. Note that the measured and modeled data points closely match for the various gradient directions illustrating the quality of the fits. The locations of the three ROIs are shown on $\bar{W}$ maps. 


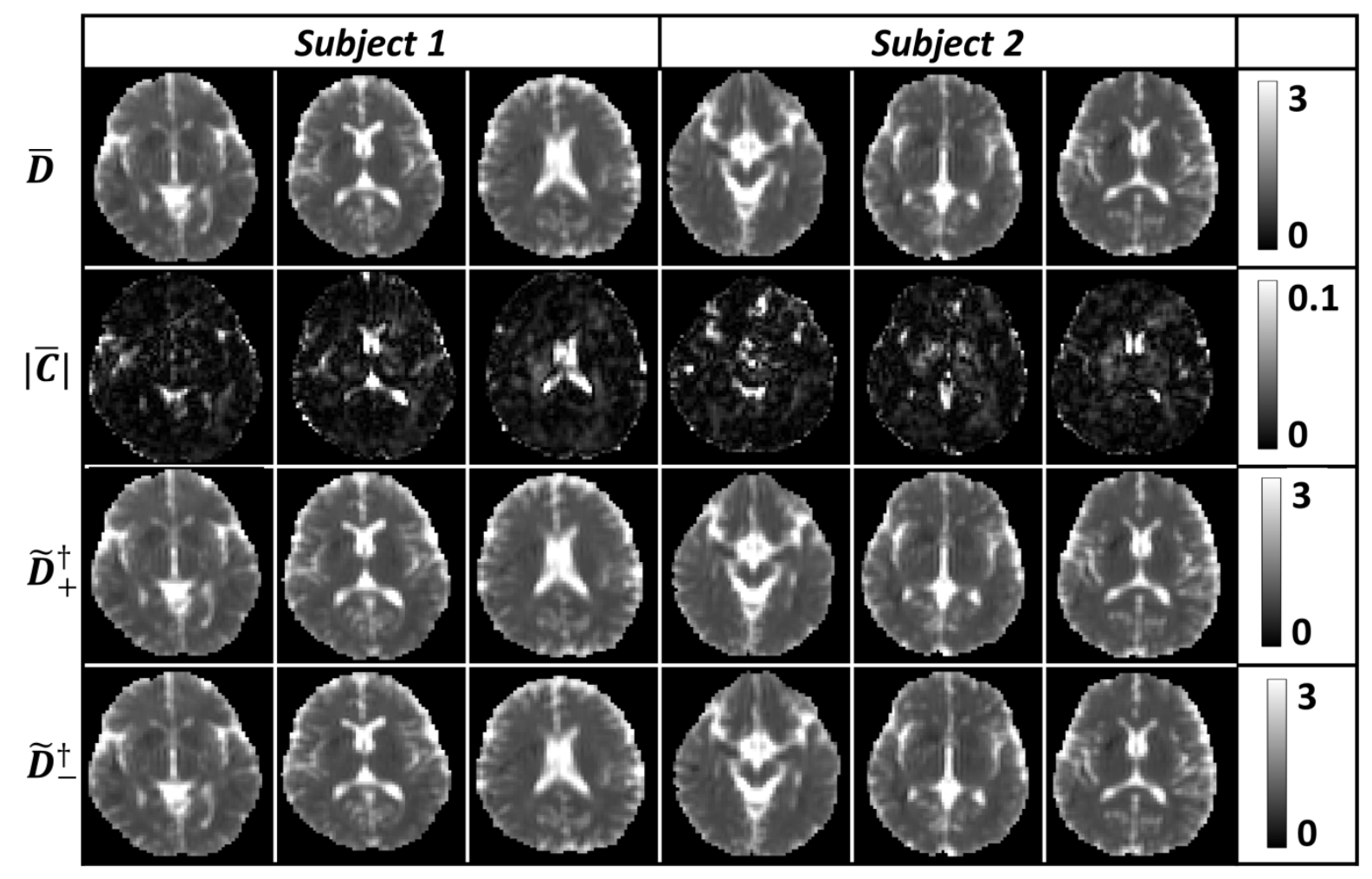

Figure 1 


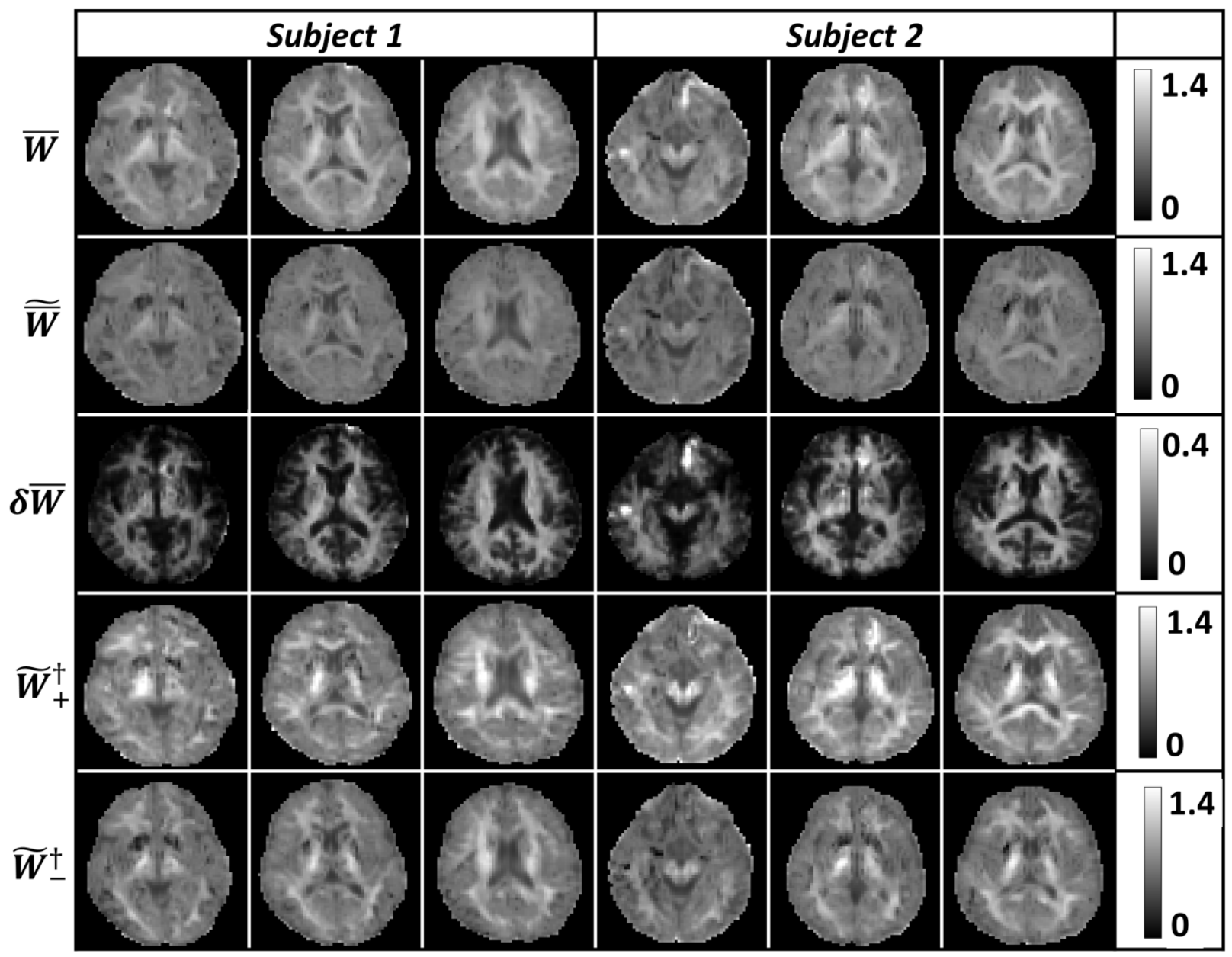

Figure 2 


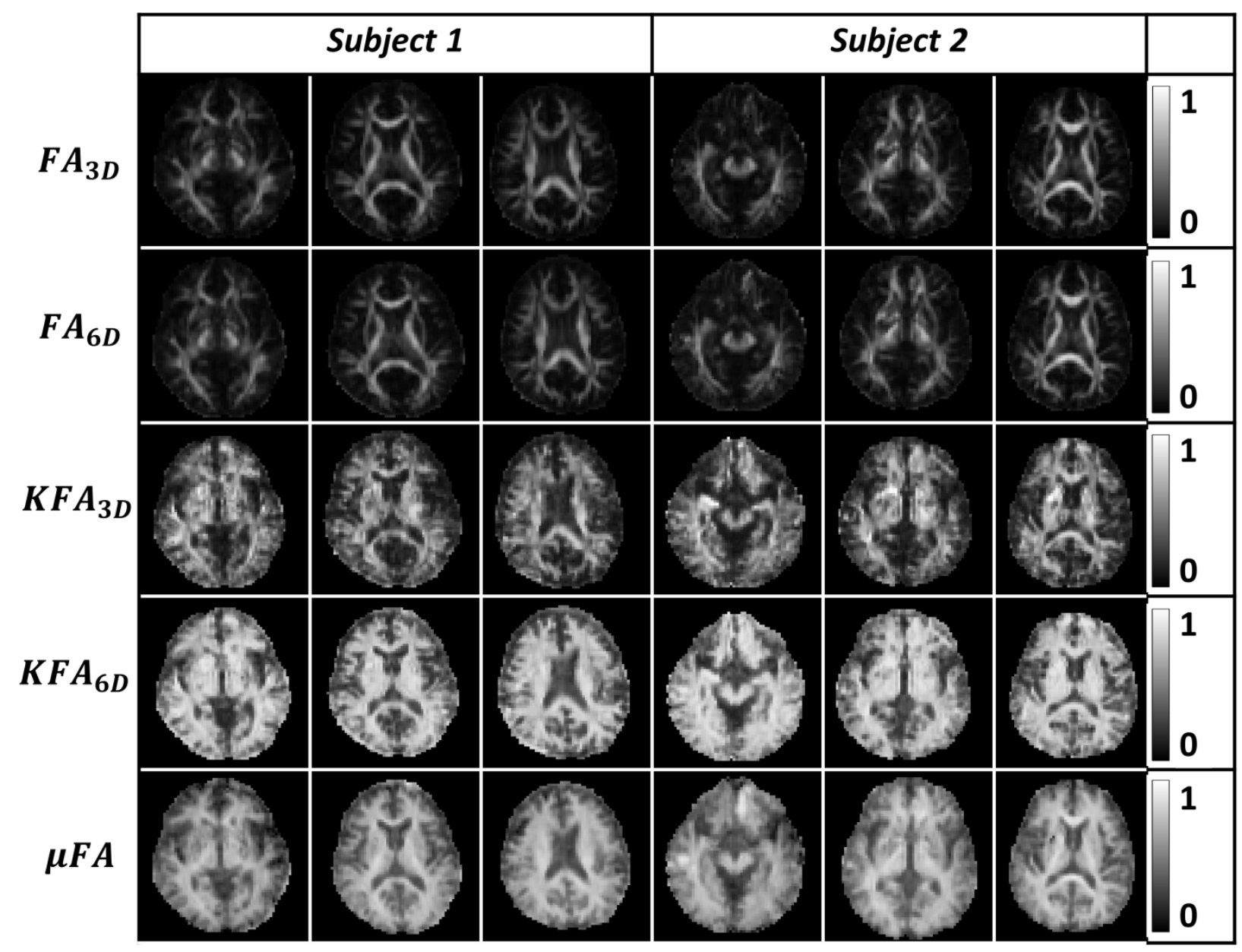

Figure 3 


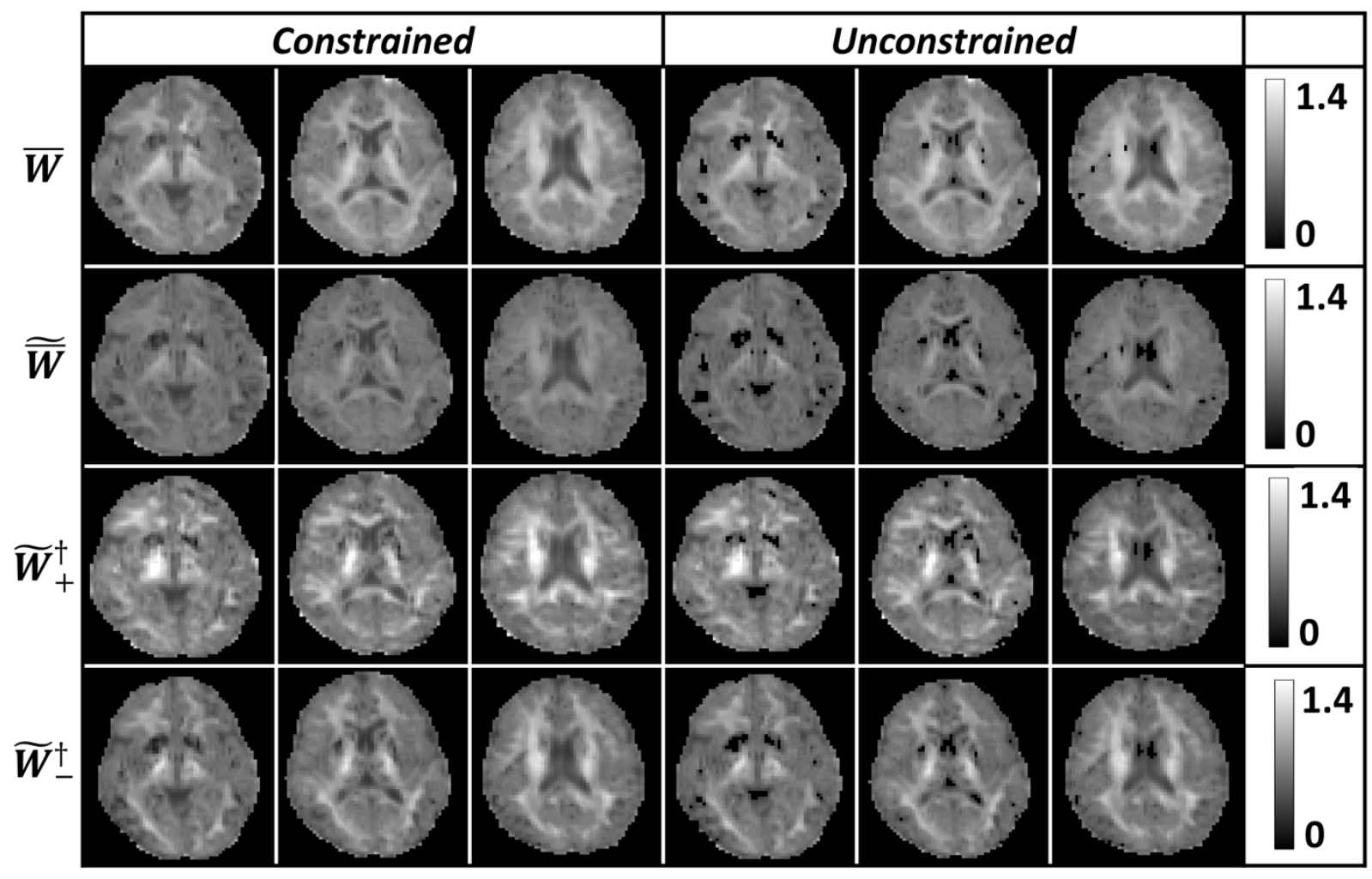

Figure 4 


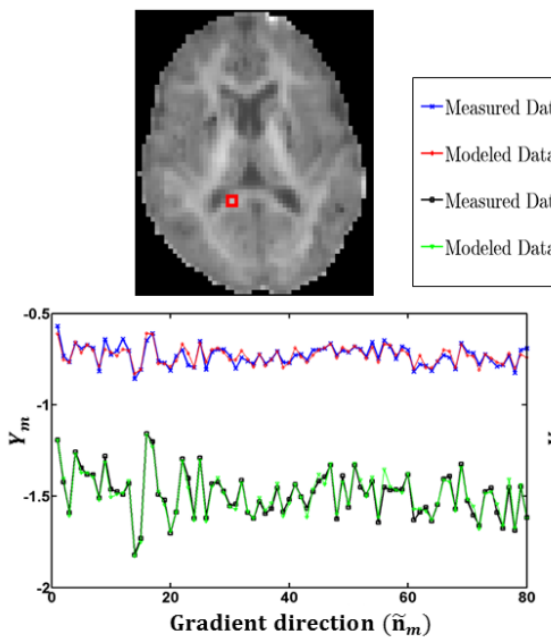

(a)
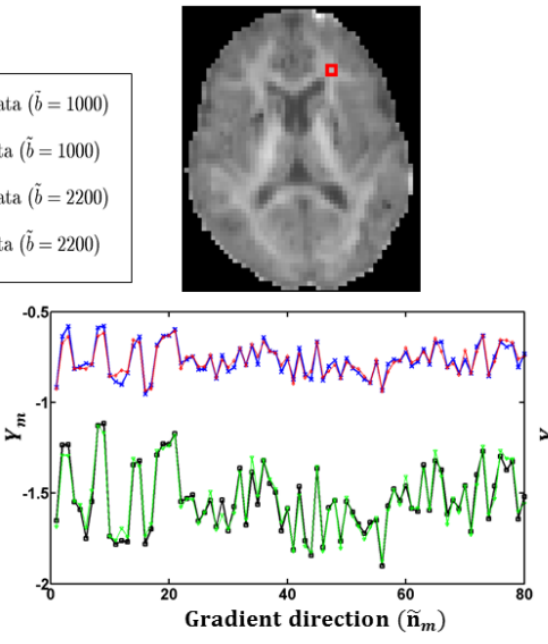

(b)
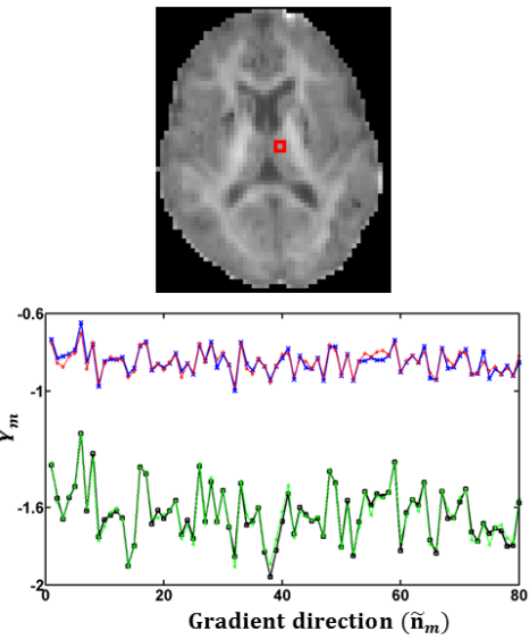

(c)

Figure 5 